\title{
Topographic Organization of the Reciprocal Connections between the Monkey Entorhinal Cortex and the Perirhinal and Parahippocampal Cortices
}

\author{
W. A. Suzuki ${ }^{1, a}$ and D. G. Amaral ${ }^{2}$ \\ ${ }^{1}$ Group in Neurosciences, University of California at San Diego, La Jolla, California 92093 and ${ }^{2}$ Center for Behavioral \\ Neuroscience, SUNY at Stony Brook, Stony Brook, New York 11794
}

\begin{abstract}
The perirhinal and parahippocampal cortices constitute the major sources of cortical input to the monkey entorhinal cortex. Neuropsychological studies have shown that these three cortical regions contribute in an important way to normal memory function. We have investigated the topographic and laminar organization of the reciprocal projections between the entorhinal cortex and these two adjacent cortical areas by placing anterograde and retrograde tracers in all three regions. There were three major findings. First, the perirhinal and parahippocampal cortices have distinct but partially overlapping interconnections with the entorhinal cortex. The perirhinal cortex tends to be interconnected with the rostral two-thirds of the entorhinal cortex while the parahippocampal cortex tends to be interconnected with approximately the caudal two-thirds of the entorhinal cortex. Second, the degree of reciprocity of the interconnections of the entorhinal cortex with the perirhinal and parahippocampal cortices differs. The parahippocampal/entorhinal connections have a high degree of reciprocity. In contrast, the degree of reciprocity of the perirhinal/entorhinal interconnections varies depending on the mediolateral position within the perirhinal cortex; medial portions of the perirhinal cortex exhibit a higher degree of reciprocity with the entorhinal cortex than lateral portions. Third, the projections from the perirhinal and parahippocampal cortices to the entorhinal cortex resemble a feedforward projection, while the projections from the entorhinal cortex to the perirhinal and parahippocampal cortices resemble a feedback projection pattern.
\end{abstract}

IKey words: hippocampal formation, connections, memory, parahippocampal gyrus, reciprocity, feedforward, feedback]

Neuropsychological studies in both humans and nonhuman priInates have provided convincing evidence that the hippocampal formation, including the dentate gyrus, hippocampus proper, subicular complex, and entorhinal cortex, is an important com-

\footnotetext{
Received Mar. 15, 1993; revised Aug. 6, 1993; acceptcd Aug. 13, 1993.

This work was supported, in part, by NIH Grant NS 16980 to D.G.A. and by NIMH Predoctoral Fellowship MH10033-02 to W.A.S. These studies were conducted, in part, at the California Regional Primale Research Center in Davis, California, Grant RR00169. We thank Janet Weber and Mary Ann Lawrence for excellent histological assistance and Jocelyne Bachevalier, Brian Leonard, Asla Pitkänen, and Lisa Stcfanacci for superb surgical assistance.

Correspondence should be addressed to Dr. David G. Amaral, Center for Behavioral Neuroscience, SUNY at Stony Brook, Stony Brook, NY 11794.

a Present address: Laboratory of Neuropsychology, NIMH, Building 49, Room 1880, 9000 Rockville Pike, Bethesda, MD 20892.
}

Copyright $\odot 1994$ Society for Neuroscience $0270-6474 / 94 / 141856-22 \$ 05.00 / 0$ ponent of the medial temporal lobe memory system (Scoville and Milner, 1957; Mishkin, 1978; Zola-Morgan and Squire, 1986). Damage to the hippocampal formation in both monkeys and humans produces an enduring (Milncr et al., 1968; ZolaMorgan et al., 1989a) polysensory anterograde amnesia (Milner, 1972; Murray and Mishkin, 1984) and a temporally graded retrograde memory impairment (Squire et al., 1989; Zola-Morgan and Squire, 1990). More remote memories appear to be largely or entirely intact following hippocampal damage.

Attention has recently been focused on the contribution to memory function from cortical regions that are adjacent and anatomically related to the hippocampal formation. Previous retrograde tracing studies demonstrated that the major sources of cortical input to the monkey entorhinal cortex are the laterally and caudally adjacent perirhinal and parahippocampal cortices. These regions contribute approximately $60 \%$ of the direct cortical input to the entorhinal cortex (Insausti et al., 1987). Studies in the monkey have shown that the perirhinal and parahippocampal cortices receive convergent inputs from a variety of unimodal and polymodal association cortices in the temporal, frontal, and parietal lobes (Jones and Powell, 1970; Van Hoesen and Pandya, 1975a; Van Hoesen et al., 1975; Seltzer and Pandya, 1976; Martin-Elkins and Horel, 1992). The entorhinal cortex then conveys this polysensory information to other areas of the hippocampal formation via the perforant path ( Van Hoesen and Pandya, 1975b; Steward, 1976; Witter et al., 1989; Witter and Amaral, 1991). The entorhinal cortex also originatcs a prominent feedback connection to the perirhinal and parahippocampal cortices that project, in turn, back to the same cortical areas from which they received inputs (Van Hoesen, 1982). Thus, the perirhinal and parahippocampal cortices may be thought of as an interface for information flow between many of the unimodal and polymodal association areas of the neocortex and the hippocampal formation.

Given the important role of the entorhinal, perirhinal, and parahippocampal cortices in conveying sensory information to and from the hippocampal formation, it is not surprising that damage to these regions produces a memory impairment in monkeys (Murray and Mishkin, 1986; Zola-Morgan et al., 1989b; Gaffan and Murray, 1992; Suzuki et al., 1993) and rats (Otto and Eichenbaum, 1992). In monkeys, bilateral lesions limited to the perirhinal and parahippocampal cortices (the PRPH lesion) produce a severe anterograde memory impairment that resembles human amnesia. Like human amnesia, the deficit is severe, long-lasting, polymodal, and affects only certain kinds of memory (Zola-Morgan et al., 1989b; Suzuki et al., 1993). Taken together, these behavioral data support the notion that 
the medial temporal lobe memory system extends beyond the hippocampal formation and includes the perirhinal and parahippocampal cortices.

Although previous neuroanatomical studies have generally demonstrated that the perirhinal and parahippocampal cortices are reciprocally connected with the entorhinal cortex, little is known about the detailed topographical organization of these interconnections. Degeneration studies in the monkey carried out by Van Hoesen and Pandya (1975a) identified the perirhinal and parahippocampal cortices as areas providing direct cortical input to the entorhinal cortex. These authors emphasized that the lateral portion of the entorhinal cortex (which they labcled the prorhinal cortex) received a particularly prominent neocortical input. More recent studies using retrograde tracers (Insausti et al., 1987) confirmed the projections from the monkey perirhinal and parahippocampal cortices to the entorhinal cortex. These studies also indicated that all portions of the entorhinal cortex appeared to receive inputs from the perirhinal and parahippocampal cortices and that all portions of the perirhinal and parahippocampal cortex project to the entorhinal cortex. However, because the tracer injections in this study tended to be large, the details of the topography of these projections could not be determined.

As a component of ongoing studies of the neuroanatomical organization of the monkey perirhinal and parahippocampal cortices, we have examined the topography and laminar organization of the reciprocal projections between the entorhinal cortex and the perirhinal and parahippocampal cortices. We have addressed three principal issues in these studies. First, we determined the topography of the afferent and efferent projections of the entorhinal cortex with the perirhinal and parahippocampal cortices. Second, we examined the degree of reciprocity of the projections of the entorhinal cortex with the perirhinal and parahippocampal cortices. Third, we examined the laminar organization of the cells of origin and the distribution of termination of these reciprocal projections.

\section{Materials and Methods}

In the present study, 36 Macaca fascicularis monkeys of either sex were used. The animals weighed approximately $3 \mathrm{~kg}$ at the time of surgery. In addition, data from experiments with nine monkeys previously described by Insausti et al. (1987) were reviewed to provide additional evidence for the topographic organization of the perirhinal and parahippocampal projections to the entorhinal cortex.

Retrograde tracer studies. The retrogradely transported tracers fast blue (FB) and diamidino yellow (DY) were injected into the perirhinal or parahippocampal cortices of 12 Macaca fascicularis monkeys. In all cases, one injection each of FB and DY was placed at different rostrocaudal levels in the perirhinal or parahippocampal cortex on one side of the brain. Of those 24 dye injections, 20 injections proved useful for thesc studics. The positions of a subset of these cases are illustrated in Figure $2 B$. In addition to the FB and DY injections, the locations of 12 cases containing retrograde tracer injections (mainly wheat germ agglutinin-conjugated horseradish peroxidase) in the entorhinal cortex are also indicated on Figure $2 B$. Nine of these cases have been described in detail elsewhere (Insausti et al., 1987).

Anterograde tracer studies. ${ }^{3} \mathrm{H}$-amino acids were injected into the perirhinal, parahippocampal, or entorhinal cortex of 27 Macaca fascicularis monkeys (the locations of these injections are plotted on the unfolded map illustrated in Fig. $2 A$ ). Five of the animals that received ${ }^{3} \mathrm{H}$-amino acid injections also received retrograde tracer injections into the perirhinal or parahippocampal cortex of the ipsilateral hemisphere.

Surgical, injection, and histological procedures. For experiments conducted prior to August 1990, animals were preanesthetized with ketamine hydrochloride ( $8 \mathrm{mg} / \mathrm{kg}$, i.m.) followed by Nembutal ( $25 \mathrm{mg} / \mathrm{kg}$, i.p.) and mounted in a Kopf stereotaxic apparatus. A venous catheter was placed and Nembutal was supplemented as necessary throughout sur- gery. For the remaining experiments, animals were preanesthetized with ketamine hydrochloride $(8 \mathrm{mg} / \mathrm{kg}$, i.m.), intubated with a tracheal cannula, and mounted in a Kopf stereotaxic apparatus. The animals were then placed on a mechanical ventilator where a surgical level of anesthesia was induced with isoflurane. Using sterile procedures, the skull was exposed and a small burr hole was made at a site appropriate for the injection as determined from the atlas of Szabo and Cowan (1984). In most cases, a tungsten microelectrode was lowered through the intended injection site and extracellular multi- and single-unit responses were recorded along its trajectory. This procedure allowed us to determine more precisely the appropriate dorsoventral coordinate for placement of the injection. In most cases, the tracer substances were dispensed through glass micropipettes using air pressure pulses (Amaral and Price, 1983). Injections aimed at the rostral tip of the temporal pole were made using a $1 \mu \mathrm{l}$ Hamilton syringe. Following injection of the tracer, the pipette or syringe was slowly withdrawn and the opening sutured. Analgesics $(0.15 \mathrm{mg} / \mathrm{kg}$ of oxymorphone given threc times daily for $2 \mathrm{~d})$ were administered immediately postsurgically and a prophylactic regime of antibiotics ( $50 \mathrm{mg} / \mathrm{kg}$ of Claforan, three times daily) was administered during the first $5 \mathrm{~d}$ of the survival period.

In the cases with injections of the fluorescent retrograde tracers DY (Dr. Illing $\mathrm{GmbH}$ and $\mathrm{Co}$.) and FB (Dr. Illing $\mathrm{GmbH}$ and Co.) between 500 and $1500 \mathrm{nl}$ of a $2 \%$ DY solution or between 500 and $650 \mathrm{nl}$ of a $3 \%$ FB solution was dispensed. Both the FB and the DY solutions were dissolved in distilled water. Animals survived for approximately $14 \mathrm{~d}$ and were then deeply anesthetized and perfused transcardially with $4 \%$ paraformaldehyde in $0.1 \mathrm{M}$ phosphate buffer $(\mathrm{pH} 7.4)$. The brains were postfixed for $6 \mathrm{hr}$ in the same fixative then cryoprotected in $10 \%$ and $20 \%$ glycerol solutions in $0.1 \mathrm{M}$ phosphate buffer of $\mathrm{pH} \mathrm{7.4}$. All brains were sectioned at $30 \mu \mathrm{m}$ on a freezing, sliding microtome. For analysis of the retrograde fluorescent tracers, two adjacent series of sections were immediately mounted on slides and stored desiccated at $20^{\circ} \mathrm{C}$ until they were analyzed with a Leitz Dialux-20 microscope equipped for fluorescence microscopy. An adjacent series of sections was stained with thionin to allow determination of the cytoarchitectonic boundaries of the entorhinal, perirhinal, and parahippocampal cortices.

The ${ }^{3} \mathrm{H}$-amino acid injections in the entorhinal, perirhinal, or parahippocampal cortex consisted of a single injection of 50-100 nl of $1: 1$ mixture of ${ }^{3} \mathrm{H}$-leucine and ${ }^{3} \mathrm{H}$-proline (concentrated to $100 \mu \mathrm{Ci} / \mu \mathrm{l}$ ). Animals survived for 7-14 $\mathrm{d}$ and were then deeply anesthetized and perfused as described above. Sections were cut at either $30 \mu \mathrm{m}$ or 50 $\mu \mathrm{m}$ and processed according to the protocol of Cowan et al. (1972) for the autoradiographic demonstration of the anterogradely transported isotope.

Analysis of retrograde and anterograde material. For the cases with retrograde tracer injections in the perirhinal or parahippocampal cortex, the distribution of retrogradely labeled cells in the entorhinal cortex was analyzed using a computer-aided $\mathrm{x}, \mathrm{y}$-plotting system and the position of each retrogradely labeled cell was plotted for every section in a 1 -in16 series through the entorhinal cortex. An average of 21 sections through the entorhinal cortex were analyzed.

For the autoradiographic experiments, the distribution of anterogradely transported ${ }^{3} \mathrm{H}$-amino acids in the entorhinal or perirhinal and parahippocampal cortices was analyzed using dark-field illumination. The position and density of labeled fibers and terminals were plotted on representative camera lucida drawings of sections through the full rostrocaudal extent of the appropriate cortical areas.

Construction of two-dimensional unfolded maps. The topographic organization of projections to and from the entorhinal cortex is most easily appreciated if the distributions of labeled cells or fibers are plotted on two-dimensional unfolded maps of the entorhinal, perirhinal, and parahippocampal cortices. The procedure used to construct the unfolded maps of the entorhinal cortex has previously been described (Amaral et al., 1987). Figure 1 shows an unfolded map, including the contour lines that were used to construct the map that encompasses the surface area of the entorhinal, perirhinal, and parahippocampal cortices; coronal sections from five rostrocaudal levels that were used in the map are also illustrated to give a sense of how the unfolded map relates to a standard series of coronal sections. In order to construct these unfolded maps, line drawings were made at a magnification of $13 \times$ with a Nikon stereomicroscope from a series of Nissl-stained sections sampled at 480 $\mu \mathrm{m}$ intervals. The boundaries of the major cortical areas were microscopically detcrmined and marked onto the line drawings, and the sections were "unfolded" using the contour lines shown in Figure 1 according to the procedure of Van Essen and Maunsell (1980). Sections 
Figure 1. A, View of the ventral surface of the brain of $M$. fascicularis. Shaded area indicates the location of the entorhinal, perirhinal, and parahippocampal cortices that have been unfolded in $B . B$, An unfolded two-dimensional map of the surface area of the entorhinal (areas $E_{O}, E_{R}, E_{L}, E_{l}, E_{C}$, and $E_{C}$ of Amaral et al., 1987), perirhinal (areas $35,36 d, 36 \mathrm{rm}, 36 \mathrm{rl}, 36 \mathrm{~cm}$, $36 \mathrm{cl}$ ), and parahippocampal (areas $T H r$, $T H c, T F m, T F l$ ) cortices with contour lines used to unfold the map. Dashed line represents the rhinal sulcus. Line drawings of representative coronal sections adapted from the atlas of Szabo and Cowan (1984) are shown at right. Arrows indicate the approximate region of the unfolded map at which the section is located. Shaded area in each of the representative coronal sections indicates the area that was unfolded in the map. The designations A25.0, A20.0, and so on, specify stereotaxic distances in millimeters anterior $(A)$ to the interaural line. Areas $T E$ and $T E O$ form the lateral border of the perirhinal and parahippocampal cortices and area $V T F$ forms the caudal border of the parahippocampal cortex. $r s$, rhinal sulcus; $P a S$, parasubiculum; $R$, rostral; $C$, caudal; $M$, medial; $L$, lateral. Scale bar, 2 mm (applies only to the unfolded map).

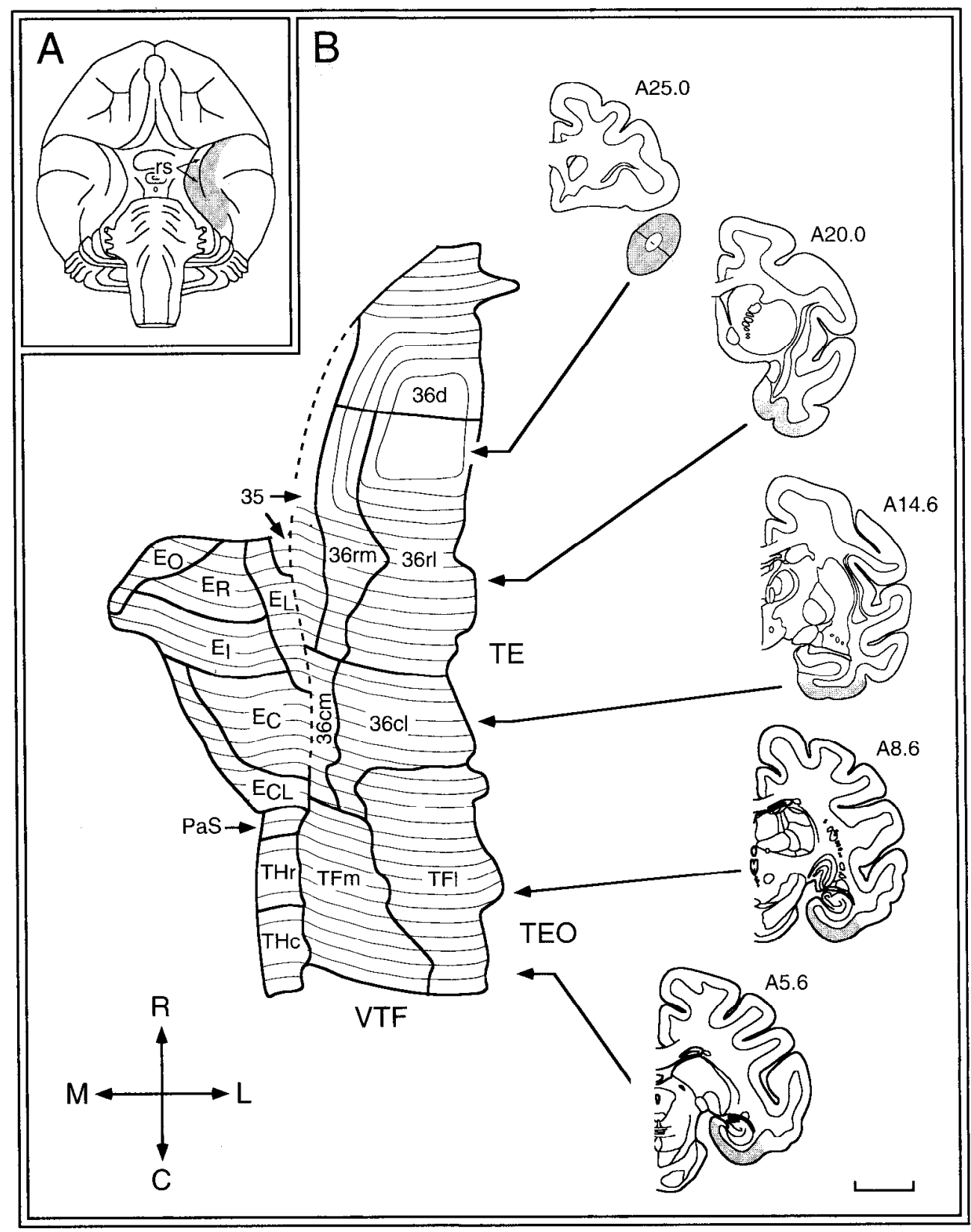

from rostral levels were aligned along the fundus of the rhinal sulcus while those from caudal levels were aligned along the border between the parahippocampal cortex and the subicular complex or retrosplenial cortex. An average of 38 sections were used to construct each of these unfolded maps.

Figures 4 and 10 show a series of unfolded maps of the entorhinal cortex indicating quantitatively the density of retrogradely labeled cells observed throughout this region following injections of tracers into different portions of the perirhinal or parahippocampal cortex. To construct the density maps, the numbers of retrogradely labeled cells in layers $V$ and VI located in contiguous $440-\mu \mathrm{m}$-wide columns along the mediolateral (transverse) extent of the entorhinal cortex were counted in a 1 -in-16 series of computer-generated digitized plots through the entorhinal cortex printed out at a magnification of $25 \times$. Since the absolute number of labeled cells in the entorhinal cortex varied from case to case (range of highest number of cells per voxel: case M-21-91 FB, 60 cells/voxel; case M-15-91 DY, 11 cells/voxel); we normalized the density of retrogradely labeled cells by first determining the number of cells in the most densely labeled column in the entorhinal cortex. This number was then divided by 4 to determine the four density levels for that case. Thus, in the unfolded density maps of the entorhinal cortex, black voxels represent regions containing the highest quartile of retrogradely labeled cells and the three progressively lighter shading patterns represent the three progressively lower quartiles of cell density. White voxels represent areas where no retrogradely labeled cells were observed. In two cases, the highest number of retrogradely labeled cells in one column was so low that using four different density levels seemed inappropriate. In case M-5-91 FB, the highest number of cells per voxel was seven. For this case, voxels with between one and four cells were shown in the lightest shading pattern and voxels with between five and seven cells were shown in the next darkest shading pattern (see Fig. 10). In case M-1-92 DY, the highest number of cells per voxel was three and all voxels containing labeled cells were shown in the lightest shading pattern (see Fig. 10).

\section{Results}

\section{Nomenclature}

The entorhinal, perirhinal, and parahippocampal cortices surround the rhinal sulcus on the ventromedial surface of the monkey brain. The terminology for the various subdivisions of these 


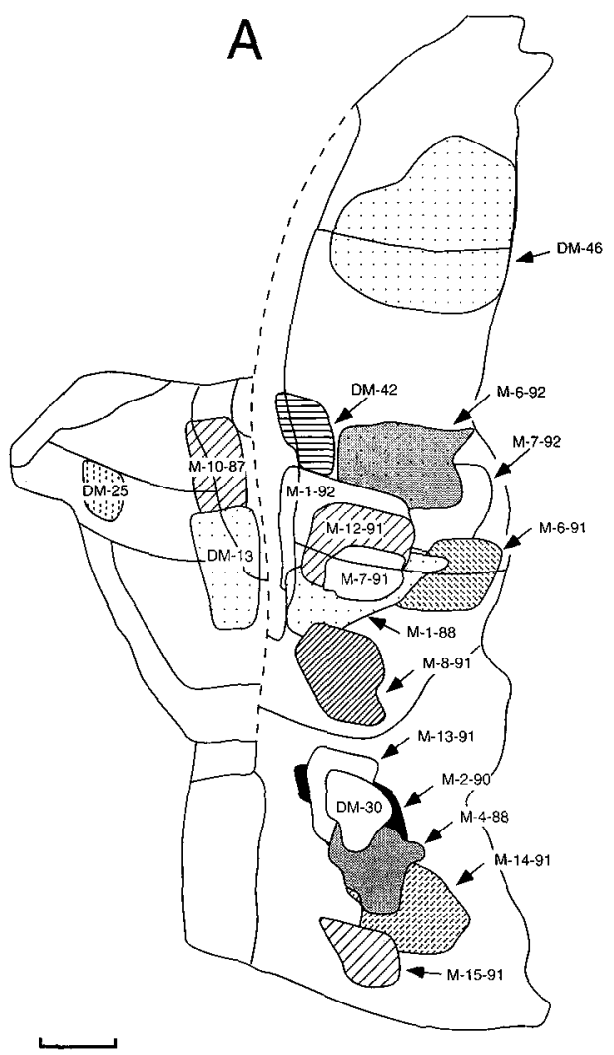

Figure 2. Two unfolded two-dimensional maps of the surface area of the entorhinal, perirhinal, and parahippocampal cortices similar to the one illustrated in Figure 1. A shows the location of the ${ }^{3} \mathrm{H}$-amino acid injections analyzed for this study. $B$ shows the locations of the retrograde tracer injections used for this study. All retrograde tracer injections in the entorhinal cortex except cases M-1-90 FB, M-11-90 DY, and M-11-90 FB have been previously presented in Insausti et al. (1987). Scale bar, $2 \mathrm{~mm}$. regions of cortex as well as the criteria used for establishing the boundaries between the subdivisions have been described previously (Amaral et al., 1987; Insausti et al., 1987). Briefly, the entorhinal cortex is subdivided into six parts that are arranged from rostral to caudal (Fig. 1). Rostrally and medially is the olfactory division $\left(E_{\mathrm{o}}\right)$, so named because it is the only entorhinal field that receives a direct projection from the olfactory bulb. $E_{\mathrm{O}}$ is bordered laterally by the rostral subdivision of the entorhinal cortex $\left(E_{R}\right)$. Area $E_{R}$, in turn, is bordered laterally by the lateral subdivision of the entorhinal cortex $\left(E_{L}\right)$. Area $E_{L}$ was originally divided into rostral and caudal divisions (Amaral et al., 1987) but is treated here as a single region. Both $E_{O}$ and $E_{R}$ are bordered caudally by the intermediate subdivision of the entorhinal cortex $\left(E_{1}\right)$. Finally, the caudal portion of the entorhinal cortex is made up of the larger, caudal division $\left(\mathrm{E}_{\mathrm{C}}\right)$, and a smaller, caudal limiting division $\left(E_{C L}\right)$.

The perirhinal cortex is made up of a smaller medially situated area 35 and a larger laterally situated area 36 . For most of its rostrocaudal extent, area 35 is confined to the fundus and lateral bank of the rhinal sulcus; only at the extreme rostral pole of the entorhinal cortex does area 35 extend slightly onto the medial bank of the rhinal sulcus. Area 35 is an agranular cortex that is characterized by a densely populated layer $\mathrm{V}$ made up of large darkly staining cells, often separated from a meagerly populated layer III by a cell-free zone. Area 36 is located just lateral to area 35 . Five subdivisions of area 36 have been recognized. At the most rostral and dorsal extent of the perirhinal cortex is area $36 \mathrm{~d}$ (the dorsal subdivision of area 36), which makes up approximately the dorsal one-third of what is typically referred to as the temporal pole. This area shares many of the same cytoarchitectonic characteristics with the other subdivisions of area 36 , but tends to be less organized and less laminated than the other subdivisions. Caudally adjacent to area $36 \mathrm{~d}$ is area $36 \mathrm{r}$ (rostral subdivision of area 36). We have further subdivided area $36 \mathbf{r}$ into $36 \mathrm{rm}$ (rostromedial subdivision of area 36 ) and area $36 \mathrm{rl}$ (rostrolateral subdivision of area 36 ). Area $36 \mathrm{rm}$ is a rather narrow cortical area that is situated lateral to area 35 , and medial to the full rostrocaudal extent of area $36 \mathrm{rl}$. This area is characterized by prominent clumps of darkly staining small cells in layer II, large lightly staining roundish cells in layer III, and large darkly staining fusiform-shaped cells in the deep layers. Area 36rl is the largest of the subdivisions of area 36. At its most rostral and dorsal extent, it makes up approximately the ventral two-thirds of what is typically referred to as the temporal pole, or area TG of von Bonin and Bailey (1947). It is bounded laterally by the unimodal visual area TE. More ventrally, area $36 \mathrm{rl}$ is adjacent to approximately the rostral half of the entorhinal cortex. Area 36 can be distinguished from the laterally adjacent area TE because the latter has a clear separation between layers $\mathrm{V}$ and $\mathrm{VI}$, and layer II is thicker and lacks the patches of darkly stained cells observed in area 36 . The cortex of area TE also has a more columnar organization. The caudal extreme of the perirhinal cortex has been called area $36 \mathrm{c}$ (caudal subdivision of area 36). We have further subdivided this area into area $36 \mathrm{~cm}$ (caudomedial subdivision of area 36 ) and area $36 \mathrm{cl}$ (caudolateral subdivision of area 36). Areas $36 \mathrm{~cm}$ and $36 \mathrm{cl}$ are medially adjacent to the intermediate and caudal divisions of the entorhinal cortex (areas $E_{1}$ and $E_{C}$ ) and are typically bounded laterally by the most rostral portion of area TF of the parahippocampal cortex. In general, these subdivisions are the most laminated and differentiated of all the subdivisions of the perirhinal cortex. Layer IV tends to be thicker in these subdivisions and the cortex has a more prominent radial organization. For clarity, we have only indicated the boundaries 
of areas 35, 36d, 36r, and $36 \mathrm{c}$ in the unfolded maps of Figures 2,5 , and 12 .

The parahippocampal cortex is caudally adjacent to the perirhinal cortex and is made up of a smaller, medially situated area $\mathrm{TH}$ and a larger, laterally situated area TF. Area $\mathrm{TH}$ has been subdivided into area $\mathrm{THr}$ (rostral subdivision of area $\mathrm{TH}$ ) and area $\mathrm{THc}$ (caudal subdivision of area $\mathrm{TH}$ ). Area $\mathrm{THr}$ is an agranular cortex with a distinctive deep cell layer V/VI made up of large and darkly staining cells. Layers II and III of area TIIr are thin, and there is no clcar border between them. Area $\mathrm{THc}$ can be distinguished from area THr by the presence of a layer IV, more radially oriented cells, and an overall more highly laminated appearance. Area TF is a larger area that is laterally adjacent to area $\mathrm{TH}$. We have subdivided area TF into areas TFm (medial subdivision of area TF) and TFl (lateral subdivision of area TF). In general, area TF is a dysgranular cortex that is distinguished by large, darkly staining cells in layers $\mathrm{V}$ and VI. Area TFm can be distinguished from area TFl because it is thinner, the cells of layer III do not show as distinctive a size gradient, and there is less of a differentiation between layers $\mathrm{V}$ and VI. Area TFl can be distinguished from the laterally adjacent areas TE or TEO because the deep cells of area TE are smaller, the cortex is much more radially organized, and layer IV becomes more prominent in area TE. Caudally, area TF is bounded by area VTF (Gattas et al., 1985). For clarity, we have only indicated the boundaries for areas TH and TF on the temporal lobe unfolded maps in Figures 2, 5, and 12.

\section{Overview of the presentation of results}

In this article we describe the topographical and laminar organization of the reciprocal projections of the monkey entorhinal cortex with the perirhinal versus the parahippocampal cortex. Because there were substantial differences in the organization of the projections of the entorhinal cortex with the perirhinal and parahippocampal cortices, we first describe the topographical organization of the reciprocal projections of the entorhinal cortex with the perirhinal cortex and then discuss the connections with the parahippocampal cortex. In the perirhinal cortex section, we start by describing a series of anterograde tracer experiments that summarize the projection from the perirhinal cortex to the entorhinal cortex. We then review a series of experiments with retrograde tracer injections into the entorhinal cortex that confirm the pattern demonstrated with the anterograde tracer experiments. Next we describe the topographical organization of the projections from the entorhinal cortex back to the perirhinal cortex. Results from a series of experiments with retrograde tracer injections into the perirhinal cortex are described along with results from complementary expcriments with antcrograde tracer injections in the entorhinal cortex. In the final two sections on the perirhinal cortex we evaluate the degree of reciprocity of the projections between the entorhinal cortex and the perirhinal cortex, and we conclude by describing the laminar organization of the reciprocal projections of the entorhinal cortex with the perirhinal cortex. In the second half of Results, we present a series of similarly organized descriptions that summarize the relationships between the entorhinal cortex and the parahippocampal cortex.

\section{Organization of the reciprocal projections of the entorhinal} cortex with the perirhinal cortex

Projections from the perirhinal cortex to the entorhinal cortex Anterograde tracer experiments. The locations of 10 representative injections of ${ }^{3} \mathrm{H}$-amino acids in the perirhinal cortex are illustrated in Figure $2 A$. The patterns of terminal labeling in the entorhinal cortex resulting from these injections are shown in a series of two-dimensional unfolded maps in Figure 3. The first feature of the projection pattern that should be noted is that all cases with ${ }^{3} \mathrm{H}$-amino acid injections in the perirhinal cortex resulted in labeling throughout an extensive rostrocaudal area of the entorhinal cortex. Moreover, even a casual survey of Figure 3 reveals one of the most striking findings of these studies; injections in all regions of the perirhinal cortex produced similar patterns of termination in the entorhinal cortex. While the general pattern of terminal labeling was similar for these cases, there were nonetheless variations in the relative regional strengths of the projections.

In order to describe the organization of these projections in more detail, we will summarize a few representative cases in two ways. We first examine the pattern of terminal labeling in the entorhinal cortex resulting from injections at three different rostrocaudal levels of the perirhinal cortex. We then examine the pattern of terminal labeling in the entorhinal cortex resulting from injections located at approximately the same rostrocaudal level of the perirhinal cortex but positioned at different mediolateral locations. Finally, we highlight a region of the perirhinal cortex that appears to project less strongly to the entorhinal cortex.

Experiments DM-46, M-7-91, and M-8-91 had ${ }^{3} \mathrm{H}$-amino acid injections at three nonoverlapping rostrocaudal levels of the perirhinal cortex (Fig. 2A). The injection in case DM-46 involved a large mediolateral extent of area $36 \mathrm{~d}$ and the rostral portion of area $36 \mathrm{r}$, the one in case M-7-91 involved the midmediolateral portion of area $36 \mathrm{r}$ slightly more caudally, and the one in case M-8-91 involved the mid-mediolateral portion of area $36 \mathrm{c}$. In cases DM-46 and M-7-91, heavy terminal labeling was observed throughout approximately the lateral two-thirds to three-fourths of the rostral entorhinal cortex. Heavy to moderate terminal labeling continued caudally in the entorhinal cortex in areas $E_{L}$ and in the lateral portions of areas $E_{l}, E_{C}$, and $E_{C L}$. In case $M-8-91$, the overall pattern of labeling was similar

Figure 3. A series of two-dimensional unfolded maps of the entorhinal cortex indicating the distribution and density of terminal labeling after $10{ }^{3} \mathrm{H}$-amino acid injections in the perirhinal cortex. Shown on the top right is an unfolded two-dimensional map (similar to the one in Fig. 1) of the entorhinal, perirhinal, and parahippocampal cortices showing the locations of the anterograde tracer injections (Fig. $2 A$ ). For each map, black areas represent the region where the heaviest terminal labeling was observed, the two progressively lighter shades of gray indicate the regions where progressively lighter terminal labeling was observed. White areas represent regions where no terminal labeling was observed. The unfolded entorhinal maps in this figure are oriented with rostral $(R)$ toward the top, caudal $(C)$ toward the bottom, medial $(M)$ toward the left of the page, and lateral $(L)$ toward the right of the page. Solid lines represent the boundaries of the different subdivisions of the entorhinal cortex. See Materials and Methods for more details on the construction of these density maps. The position of the unfolded entorhinal maps on the page corresponds to the relative locations of their injection sites within the perirhinal cortex as shown in Figure $2 A$. Thus, the unfolded maps positioned toward the top, bottom, left, or right of the page had injections situated in the rostral, caudal, medial, or lateral portions of the perirhinal cortex, respectively. Dashed line represents the rhinal sulcus. Scale bar, $2 \mathrm{~mm}$. 

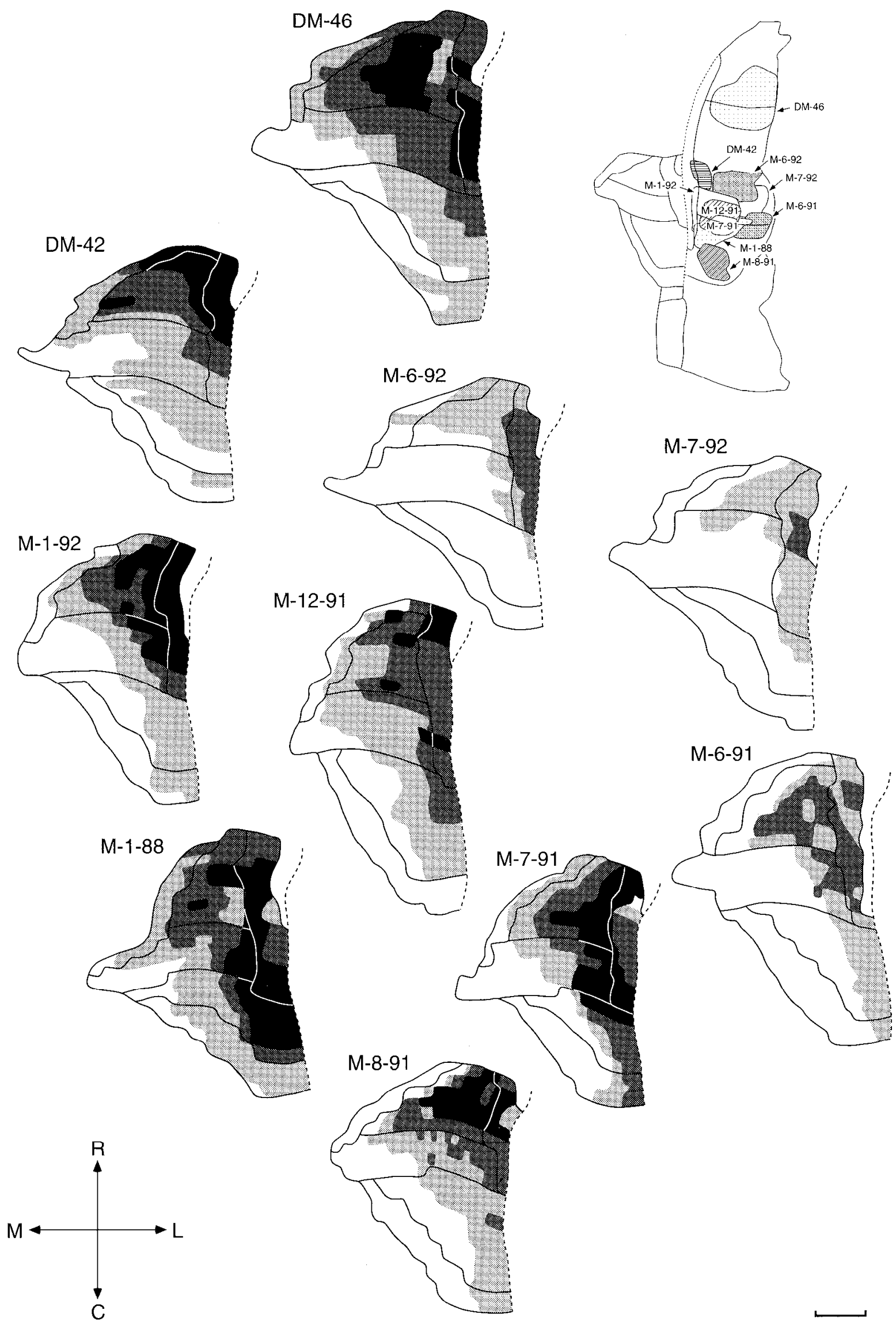


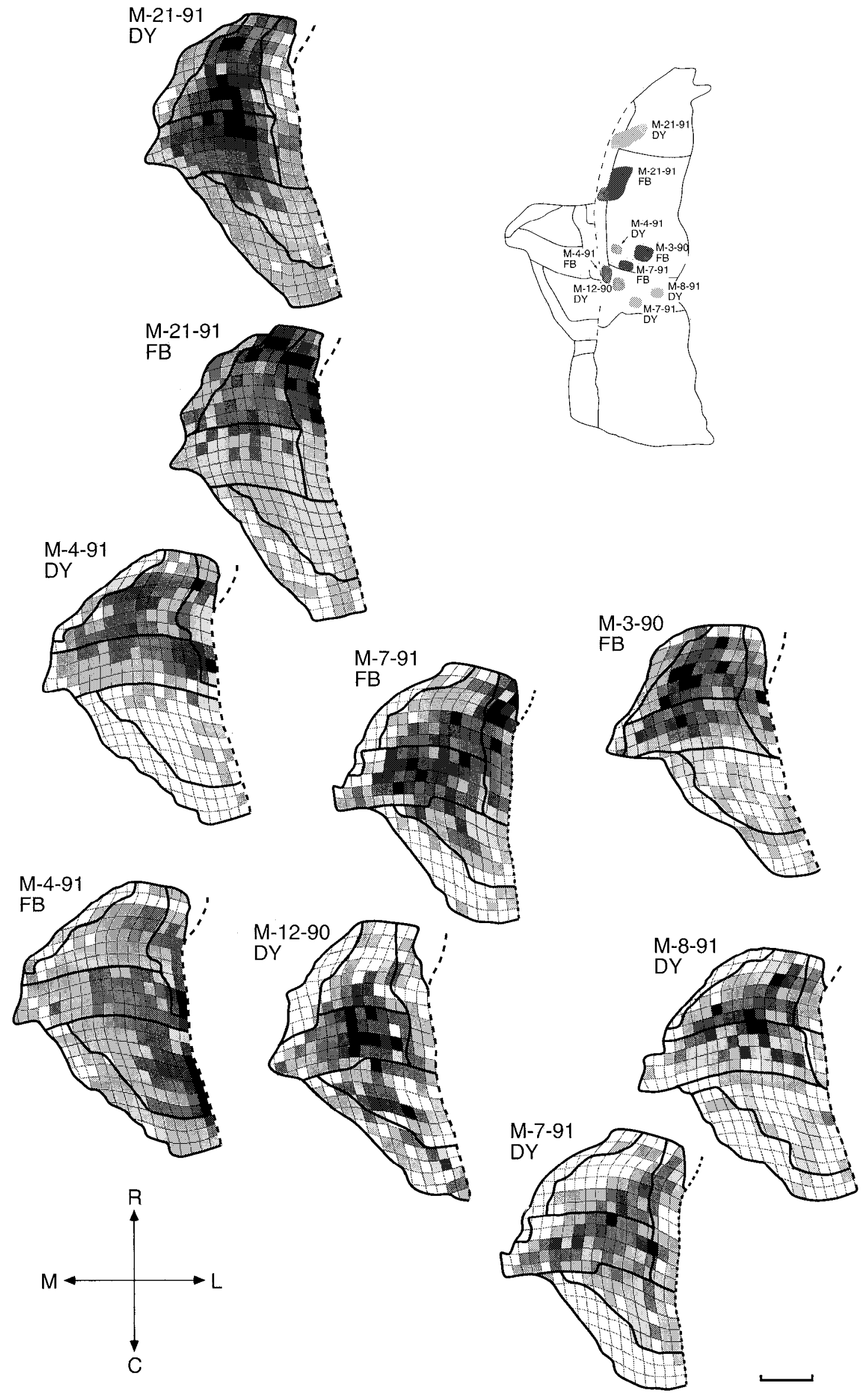


to the previous two cases but the density distribution of terminal labeling differed slightly. There was heavy terminal labeling in the lateral two-thirds of the rostral portion of the entorhinal cortex, but this did not continue as far caudally as in the other cases; only weak terminal labeling was observed in the lateral aspects of areas $\mathrm{E}_{C}$ and $\mathrm{E}_{\mathrm{CL}}$

The organization of projections from different mediolateral portions of the perirhinal cortex to the entorhinal cortex can be evaluated by examining cases M-1-92, M-7-91, and M-6-91 in Figure 3. The injection in experiment M-1-92 involved the lateral aspect of area 35 and the medial two-thirds of area 36 . The injection in experiment M-7-91 involved the mid-mediolateral portion of area 36 and the one in experiment M-6-91 involved the lateral aspect of area 36. Despite the different mediolateral locations of these injections, the areas of terminal labeling in the entorhinal cortex were essentially the same (Fig. 3); the strongest terminal labeling in each case was located rostrally and laterally in the entorhinal cortex.

Three of the experiments illustrated in Figure 3 demonstrated relatively low levels of terminal labeling and should be considered separately. The injection in experiment M-6-92 was located at a mid to lateral region of the rostral area 36r. Cytoarchitectonically, this region is indistinguishable from the rest of area 36 , but as demonstrated here as well as in our retrograde tracer studies (Fig. 15c of Insausti et al., 1987), it generates a relatively weak projection that is confined to the lateral portion of the rostral entorhinal cortex (Fig. 3). The projections demonstrated in experiments M-7-92 and M-6-91 were also meager. The injections in these cases were located at the lateral border of area $36 \mathrm{r}$ and at the lateral border at the transition between area $36 \mathrm{r}$ and $36 \mathrm{c}$, respectively. This lateral region exhibits both cytoarchitectonic and immunohistochemical characteristics that appear to be "transitional" between area 36 and the laterally adjacent unimodal visual area TE. Area TE does not project directly to the entorhinal cortex (Insausti ct al., 1987), and thus the weaker projection observed in experiments M-7-92 and M-891 perhaps reflects a gradual change from perirhinal-like to TElike cortex.

To summarize, injections involving any rostrocaudal or mediolateral level of the perirhinal cortex produce a similar pattern of terminal labeling in the entorhinal cortex (see Fig. 12A). Rostrally, projections from the perirhinal cortex cover much of the mediolateral extent of the entorhinal cortex while caudally, the projections tend to be confined to the lateral portion of the entorhinal cortex. Although all regions of the perirhinal cortex appear to project in a similar fashion to the entorhinal cortex, there are variations in the strength of the projections. In particular, the rostral portions of area $36 \mathrm{r}$ and the lateral portions of areas $36 \mathrm{r}$ and $36 \mathrm{c}$ appear to originate weaker projections to the entorhinal cortex. These findings indicate that there is a very substantial convergence of information from all parts of the perirhinal cortex onto the rostral and lateral portions of the entorhinal cortex.

Retrograde tracer experiments. The conclusions from the an-
Table 1. Percentage of retrogradely labeled cells (from Insausti et al., 1987)

\begin{tabular}{lrrrrrrrrrr}
\multicolumn{1}{l}{ Cases $^{a}$} \\
\cline { 2 - 9 } Area & IM-4 & IM-6 & IM-8 & M-4-86 & IM-3 & IM-1 & IM-7 & DM-45 & IM-10 \\
\hline 35 & 64 & 32 & 18 & 2 & 1 & $<1$ & 12 & 9 & 14 \\
36 & 36 & 46 & 62 & 8 & 17 & 13 & 29 & 41 & 25 \\
Sub- & & & & & & & & & \\
$\quad$ total & 100 & 78 & 80 & 10 & 18 & 13 & 41 & 50 & 39 \\
TH & 0 & 7 & 7 & 23 & 38 & 35 & 11 & 14 & 25 \\
TF & 0 & 15 & 13 & 67 & 44 & 52 & 48 & 36 & 36 \\
Sub- & & & & & & & & \\
$\quad$ lotal & 0 & 22 & 20 & 90 & 82 & 87 & 59 & 50 & 61 \\
\hline a See Figure 2B for location of injection sites. &
\end{tabular}

terograde tracer experiments described above make some specific predictions concerning the distribution and density of retrogradely labeled cells in the perirhinal cortex resulting from tracer injections into the entorhinal cortex. These predictions were evaluated by reexamining data from the studies of Insausti et al., (1987) as well as by studying the distribution of labeled cells in the perirhinal cortex in experiments with entorhinal injections of retrograde tracers carried out after the Insausti et al. (1987) study. Figure $2 B$ shows the location of the nine entorhinal injections of retrograde tracers initially studied by Insausti et al. (1987) as well as three additional retrograde tracer studies conducted at a later date (cases M-1-90 FB, M-11-90 DY, and M-11-90 FB).

In the study by Insausti et al. (1987), the number of retrogradely labeled cells in the perirhinal (areas $35,36 \mathrm{pm}, 36 \mathrm{r}$, and 36c) and parahippocampal (areas TH and TF) cortices was determined (see Table 1 in Insausti et al., 1987), and the percentage of labeled cells within each of the major subdivisions of the perirhinal and parahippocampal cortices was calculated. The results of this analysis are reproduced as Table 1 in the present article. There is generally very good correspondence of these retrograde tracer studies with the results of the anterograde studies presented in this article. The highest percentage of retrogradely labeled cells in the perirhinal cortex was observed after injections involving the rostral (cases IM-6, IM-8, and IM-4) and caudolateral portions of the entorhinal cortex (cases IM-7, DM-45, and IM-10). The lowest percentage of retrogradely labeled cells was observed after injections in the caudal and medial aspects of the entorhinal cortex (cases M-4-86, IM-3, and IM1). As noted above, the retrograde tracer experiments in Insausti et al., (1987) also provided confirmatory evidence that the rostral portion of area $36 \mathrm{r}$ originates a weaker projection to the entorhinal cortex than other regions of the perirhinal cortex. Figure $15 C$ in Insausti et al., (1987) shows an unfoldcd map of the temporal lobe illustrating a summary of the density and distribution of labeled cells after all nine cases of retrograde tracer injection in the entorhinal cortex. Area 36r exhibited a

\section{$\leftarrow$}

Figure 4. A series of two-dimensional unfolded maps of the entorhinal cortex indicating the relative density of retrogradely labeled cells in nine different cases with retrograde tracer injections in the perirhinal cortex. At the top right is an unfolded two-dimensional map showing the relative locations of these retrograde tracer injections in the perirhinal cortex (Fig. $2 B$ ). For each map, the black voxels represent areas with the densest retrograde labeling and progressively lighter shades of gray represent progressively lower density of labeling. See Materials and Methods for more dctails on the construction of these density maps. Solid lines represent the boundaries of the different subdivisions of the entorhinal cortex. As in Figure 3, the position of the unfolded entorhinal maps on the page corresponds to the relative locations of their injection sites within the perirhinal cortex as shown in Figure $2 B$. Scale bar, $2 \mathrm{~mm}$. 

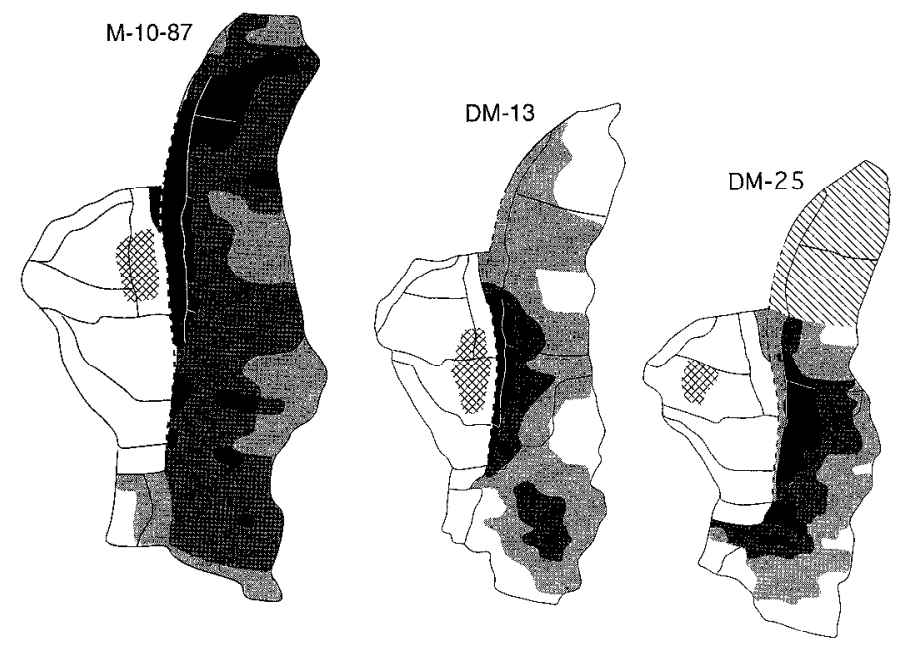

Figure 5. Two-dimensional unfolded maps of the entorhinal, perirhinal, and parahippocampal cortices in three different cases with ${ }^{3} \mathrm{H}$ amino acid injections in the entorhinal cortex. Cross-hatched area indicates the location of the injection site in the entorhinal cortex. For each map, black areas represent the region where the heaviest terminal labeling was observed, the two progressively lighter shades of gray indicate the regions where progressively lighter terminal labeling was observed. White areas represent regions where no terminal labeling was observed. Solid lines represent the boundaries of the different subdivisions of the entorhinal, perirhinal, and parahippocampal cortex. Broken line represents the rhinal sulcus. Hatching in the polar portion of the perirhinal cortex in case DM-25 represents a region where histological material was not available for analysis. Scale bar, $2 \mathrm{~mm}$.

strikingly lower number of retrogradely labeled cells compared to areas $35,36 \mathrm{c}$, and $36 \mathrm{p}$.

\section{Projections from the entorhinal cortex to the perirhinal cortex}

Retrograde tracer experiments. In order to determine the topography of projections from the entorhinal cortex to the perirhinal cortex, a series of retrograde tracer injections were placed throughout the perirhinal cortex and the distribution of retrogradely labeled cells in the entorhinal cortex was determined. Figurc $2 B$ shows the location of nine representative retrograde tracer injections in the perirhinal cortex. The relative density and distribution of retrogradely labeled cells in the entorhinal cortex resulting from these injections are illustrated in a series of two-dimensional unfolded maps (Fig. 4); increasingly higher densities of labeled cells are indicated by progressively darker shading patterns. All of the perirhinal cortex injections resulted in labeled cells throughout a large extent of the entorhinal cortex. However, in contrast to the projections from the perirhinal cortex to the entorhinal cortex, which appeared to lack any substantial topographic organization, the projections from the entorhinal cortex to the perirhinal cortex exhibited both a rostrocaudal and a mediolateral topography.

The rostrocaudal aspect of the organization of projections from the entorhinal cortex to the perirhinal cortex is rather subtle but can be appreciated by comparing experiments with retrograde tracer injections in the most rostral portions of the perirhinal cortex (cases M-21-91 DY, M-21-91 FB, and M-4$91 \mathrm{DY}$; in all cases, the initials FB refer to injections of fast blue and DY to diamidino yellow) with a case containing an injection in the caudal portions of the perirhinal cortex (cases M-7-91
DY). There is a general tendency for more cells to be labeled in rostral portions of the entorhinal cortex following injections in the rostral perirhinal cortex and more cells to be labeled at mid-rostrocaudal levels of the entorhinal cortex after injections of caudal portions of the perirhinal cortex (Fig. 4). In all cases, there was a relatively low number of labeled cells in the most caudal fields of the entorhinal cortex $\left(\mathrm{E}_{\mathrm{C}}\right.$ and $\left.\mathrm{E}_{\mathrm{CL}}\right)$.

The mediolateral organization of entorhinal projections to the perirhinal cortex can be appreciated by examination of experiments M-4-91 FB, M-12-90 DY, and M-8-91 DY (Fig. 4). The injection in experiment M-4-91 FB mainly involved area 35 (Fig. $2 B$ ). The largest number of retrogradely labeled cells was observed in the lateral and caudal portions of the entorhinal cortex (Fig. 4). The injections in experiments M-12-90 DY and M-8-91 DY involved the medial and lateral portions of area $36 \mathrm{c}$, respectively. In both cases, the largest numbers of retrogradely labeled cells were located more medially in the entorhinal cortex compared to the labeling in case M-4-91 FB. A similar pattern can be observed by comparing case M-4-91 DY and case M-3-90 FB (Fig. 4). In both experiments, injections were located at approximately the same rostrocaudal level in the perirhinal cortex but the injection in case M-4-91 DY involved the medial aspect of area 36r whereas the injection in M-3-90 FB involved a mid-mediolateral position in area $36 \mathbf{r}$. The largest number of retrogradely labeled cells in case M-4-91 DY were located rostrally and laterally in the entorhinal cortex whereas the greatest labeling in case M-3-90 FB was situated more medially in the entorhinal cortex. These data indicate that cells located laterally in the entorhinal cortex project to medial portions of the perirhinal cortex whereas cells located more medially in the entorhinal cortex project preferentially to the lateral portions of the perirhinal cortex.

To summarize, approximately the rostral two-thirds of the entorhinal cortex projects strongly to all regions of the perirhinal cortex (see Fig. 12B). There appears to be both a rostrocaudal and mediolateral topography to these projections. Whereas the rostral levels of the entorhinal cortex project to the rostral aspects of the perirhinal cortex, mid-rostrocaudal levels of the entorhinal cortex project to caudal portions of the perirhinal cortex. The mediolateral component of the topography indicates that lateral aspects of the entorhinal cortex project to the medial aspccts of the perirhinal cortex, while progressively more medial aspects of the entorhinal cortex project more laterally in the perirhinal cortex. It should be noted, however, that the most medial portions of the entorhinal cortex only generate a light to moderate projection to the perirhinal cortex.

Anterograde tracer experiments. In order to confirm the topography of projections from the entorhinal cortex to perirhinal cortex observed in the retrograde tracer experiments, a series of experiments with ${ }^{3} \mathrm{H}$-amino acid injections involving different portions of the entorhinal cortex were analyzed. The relative density and distribution of anterogradely labeled fibers and terminals in the perirhinal and parahippocampal cortices resulting from three of these experiments are shown in Figure 5. The location of these representative injections is also illustrated in Figure $2 A$.

In experiment $\mathrm{M}-10-87$, the amino acid injection involved mainly the rostral portion of area $E_{L}$. This injection produced strong labeling throughout the rostrocaudal extent of the perirhinal cortex. As would be expected from the retrograde tracer studies, the strongest labeling was observed in area 35 and in the medial part of area 36 , that is, the medial portion of the 
perirhinal cortex. There was moderate to weak labeling observed in the mid to lateral portions of area 36.

In experiment DM-13, the isotope injection involved caudal portions of $E_{L}$ as well as the lateral aspect of $E_{C}$. As in case M-10-87, the strongest labeling in DM-13 was located medially in the perirhinal cortex involving area 35 as well as the medial portion of area 36 . There was moderate to weak labeling observed throughout the mid to lateral aspects of area 36. Moreover, consistent with the topography derived from the retrograde tracer experiments, the more caudally located injection in case DM-13 tended to produce the strongest anterograde labeling more caudally in the perirhinal cortex compared to case M-10-87.

In contrast to the previous two cases, the isotope injection in experiment DM-25 was located in the medial half of $E_{1}$. As would be predicted from the retrograde studies, area 35 received only minor innervation and the strongest anterograde labeling was observed in the medial half of area 36 of the perirhinal cortex; the lateral half of area 36 was moderately to weakly labeled. A comparison of case DM-25 with case M-10-87 illustrates the mediolateral topography observed in the retrogradc tracer experiments. While the more medially placed injection in the entorhinal cortex produced the strongest labeling in the lateral portion of the perirhinal cortex (case DM-25), the more laterally placed injection in the entorhinal cortex produced the strongest labeling more medially in the perirhinal cortex (case M-10-87). Thus, the anterograde tracer injections in the entorhinal cortex confirm both the rostrocaudal and the mediolateral topography of the projections from the entorhinal cortex to the perirhinal cortex suggested by the retrograde tracer experiments.

The degree of reciprocity of projections between the entorhinal cortex and the perirhinal cortex

Analysis of individual experiments with single anterograde or retrograde tracer injections into the perirhinal cortex indicated that the bidirectional projections between the perirhinal and entorhinal cortices were organized in different ways (compare Figs. 3, 4). Moreover, because of the particular arrangement of the afferent and efferent pattern of connections, the degree of reciprocity appeared to vary depending on the region of perirhinal cortex examined.

To examine more directly the degree of reciprocity of projections from the perirhinal cortex, we conducted experiments in which pairs of anterograde and retrograde tracer injections were placed close to each other in the perirhinal cortex. One of these experiments is case M-7-91, in which the center of an anterograde tracer was located $0.48 \mathrm{~mm}$ caudal and approximately $1.23 \mathrm{~mm}$ lateral to the center of a retrograde tracer injection. Both injections were located at a mid-mediolateral portion of area 36 of the perirhinal cortex (Fig. $2 A, B$ ). Analysis of the distribution of anterograde and retrograde labeling from closely placed injections in the perirhinal cortex of the same animal provided the opportunity to map both anterograde and retrograde labeling onto the same unfolded map of the entorhinal cortex. Figure 6 shows replicas of the unfolded map from case M-7-91, with the distribution of labeled fibers and terminals on the one (Fig. $6 A$ ) and the distribution of retrogradely labeled cells on the other (Fig. $6 B$ ). Although there was obviously extensive overlap in the patterns of anterograde and retrograde labeling, there were also equally clear differences in the labeling patterns. For example, the relatively high density of retrogradely labeled cells in the medial half of $\mathrm{E}_{\mathrm{l}}$ (Fig. $6 \mathrm{~B}$ ) corresponded to
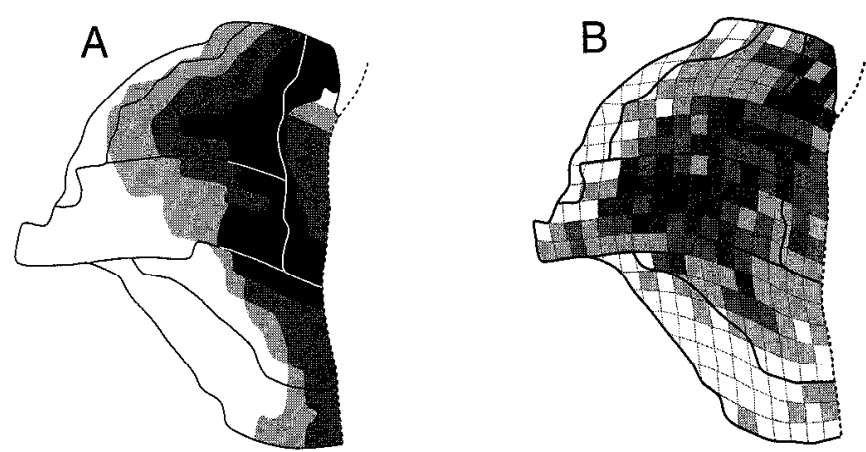

Figure 6. Two identical two-dimensional unfolded maps of the entorhinal cortex in case M-7-91. The map in $A$ illustrates the distribution of terminal labeling in the entorhinal cortex resulting from the ${ }^{3} \mathrm{H}$-amino acid injection in the perirhinal cortex (see Fig. $2 A$ ). The map in $B$ indicates the distribution and density of retrogradely labeled cells resulting from the FB injection (see Fig. $2 B$ ).

a region that received relatively meager anterograde transport from the perirhinal cortex (Fig. 6.4). Moreover, there were retrogradely labeled cells located in extreme medial portions of area $E_{1}$ that received no projection from the perirhinal cortex. The same general pattern can be seen by comparing cases M-691 , which contained an isotope injection in the lateral extreme of area 36, and case M-8-91 DY, which contained a retrograde tracer injection at approximately the same level. Although it is more difficult to compare projection patterns across animals, it is clear that these cases, like case M-7-91, did not exhibit a high degree (i.e., point to point) of reciprocity.

The degree of reciprocity of medial portions of the perirhinal cortex with the entorhinal cortex can be evaluated by comparing case $\mathrm{M}-1-88$, which contained an amino acid injection involving the medial and mid-portions of the perirhinal cortex (Fig. 3), with case M-4-91 FB, which had a retrograde tracer injection focused in area 35 at approximately the same rostrocaudal level (Fig. 4). The strongest terminal labeling in case M-1-88 was located rostrally and laterally in the entorhinal cortex; the heavy labeling continued caudally for some distance along the lateral border of the entorhinal cortex. In case $\mathrm{M}-4-91$, the highest density of retrogradely labeled cells was also located in a laterally situated region that extended for a long rostrocaudal extent of the entorhinal cortex. To summarize, the medial portions of the perirhinal cortex appear to have a higher degrce of reciprocity with the entorhinal cortex than laterally situated portions.

\section{The laminar organization of the reciprocal projections} between the entorhinal cortex and the perirhinal cortex

\section{The projection from the perirhinal cortex to the entorhinal} corlex

Cells of origin. As described by Insausti et al. (1987), cells of layer III give rise to the vast majority of the projection from the perirhinal cortex to the entorhinal cortex.

Laminar organization of termination. The distribution pattern of labeled fibers and terminals in entorhinal cortex resulting from an anterograde tracer injection in the perirhinal cortex was complex (Fig. $7 A$ ). In general, however, the strongest terminal labeling was observed in layers I, II, and the superficial portion of layer III of the cntorhinal cortex. It should be noted, however, that in the areas of heaviest projection (Fig. $7 \mathrm{~A}$, a and $\mathrm{b}$ ), all layers contained some apparent terminal labeling. 

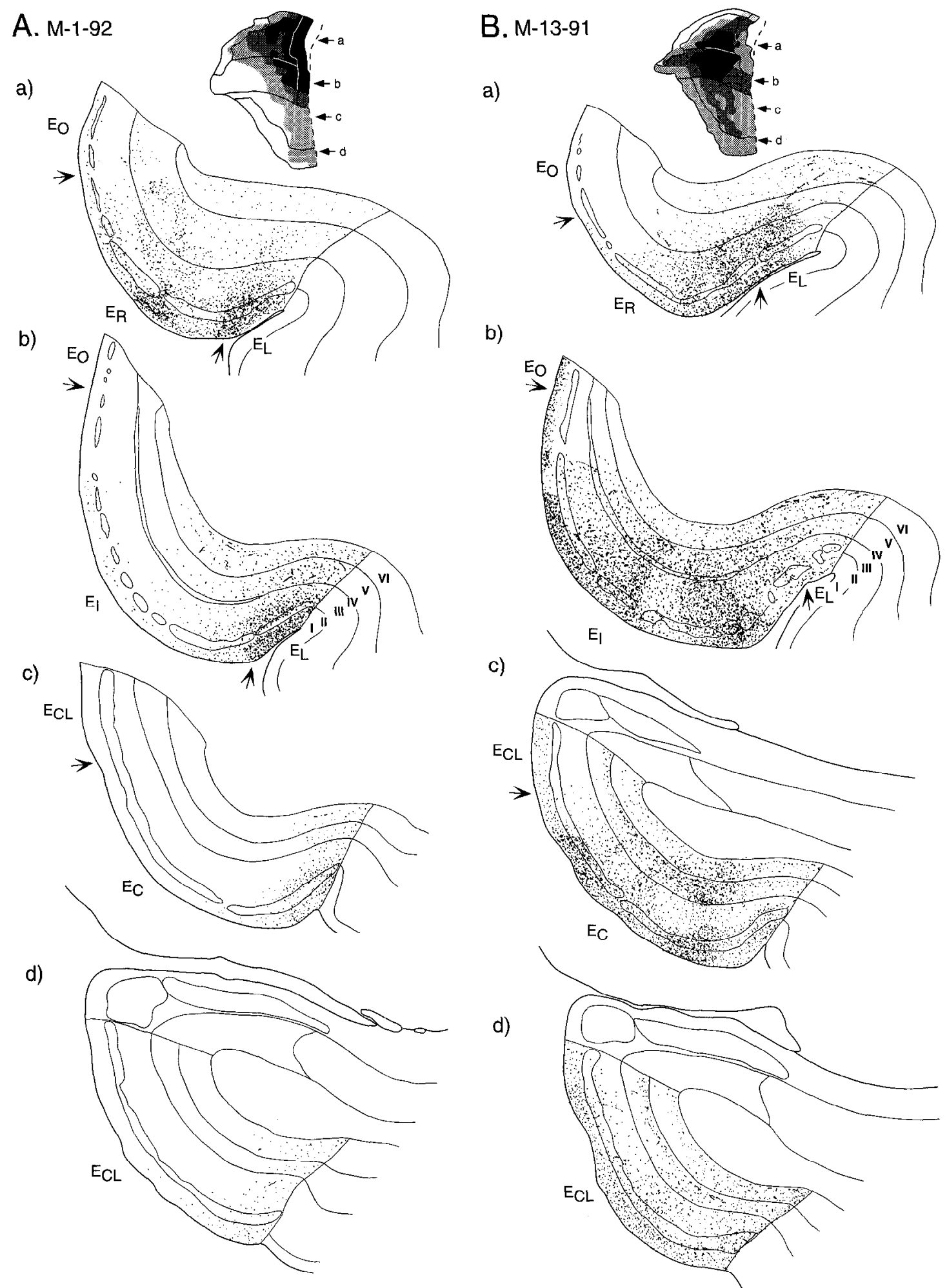

b)

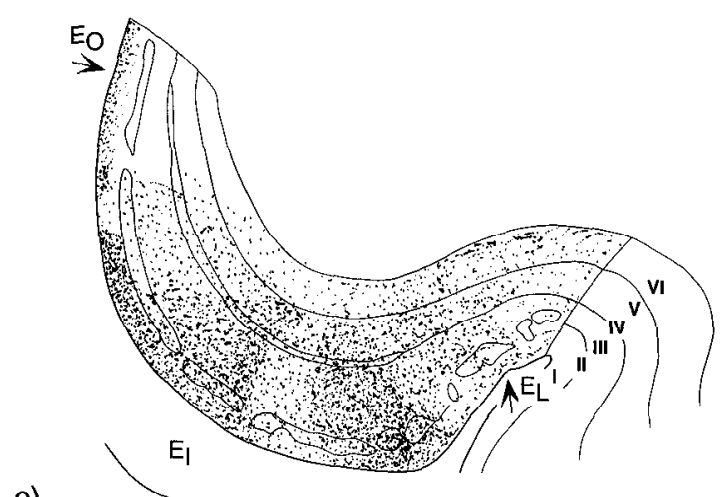

c)
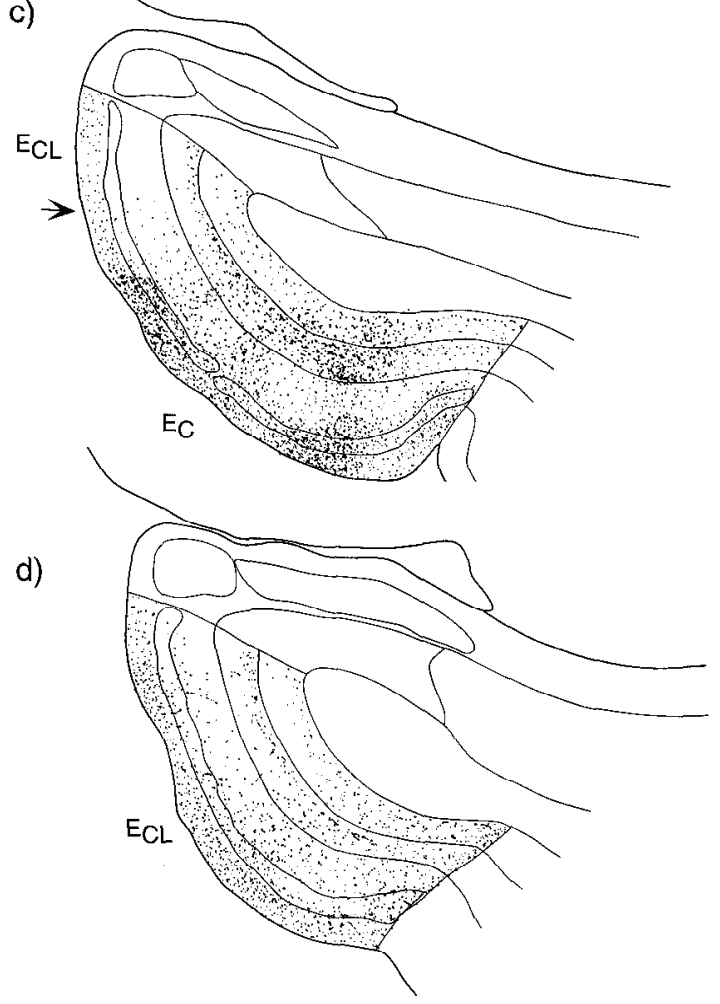

Figure 7. A, Line drawings of representative coronal sections through the entorhinal cortex arranged from rostral $(a)$ to caudal $(d)$ in case M-192 illustrating the distribution of anterograde labeling following an injection in the perirhinal cortex. Inset shows a two-dimensional unfolded map of the entorhinal cortex for that case illustrating the overall distribution of labeling. Arrows on the unfolded maps indicate the rostrocaudal levels at which the coronal sections were taken. $B$, Line drawing of representative coronal sections through the entorhinal cortex in case M-13-91 illustrating the distribution of anterograde labeling following an injection in the parahippocampal cortex. All conventions as in $A$. $I-V I$ refer to cortical layers. Scale bar, $2 \mathrm{~mm}$. 

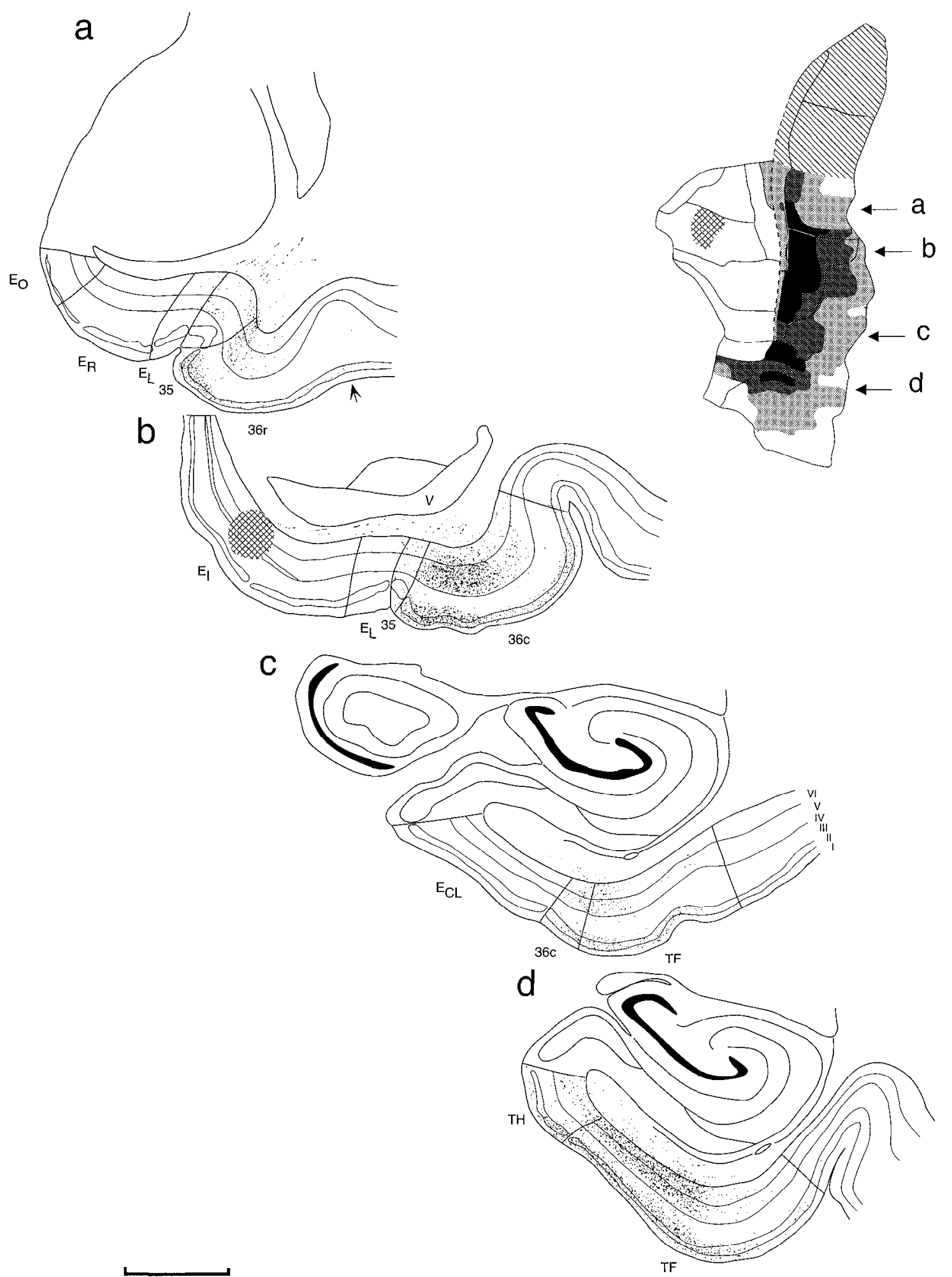

Figure 8. Line drawings of representative coronal sections through the perirhinal and parahippocampal cortices from rostral (a) to caudal (d) illustrating the distribution of anterograde labeling following an injection in the entorhinal cortex in case DM-25. Inset shows an unfolded map of the entorhinal, pcrirhinal, and parahippocampal cortices indicating the location of the injection site and the rostrocaudal levels from which the sections were taken. $V$, ventricle. Scale bar, $2 \mathrm{~mm}$.

The projection from the entorhinal cortex to the perirhinal cortex

Cells of origin. The projection from the entorhinal cortex to the perirhinal cortex originated mainly from cells situated in layer
V. Retrograde tracer injections in the perirhinal cortex also resulted in some labeled cells in layer VI and relatively fewer cells in layer III.

Laminar organization of termination. Projections from the entorhinal cortex to the perirhinal cortex terminated most heavily 

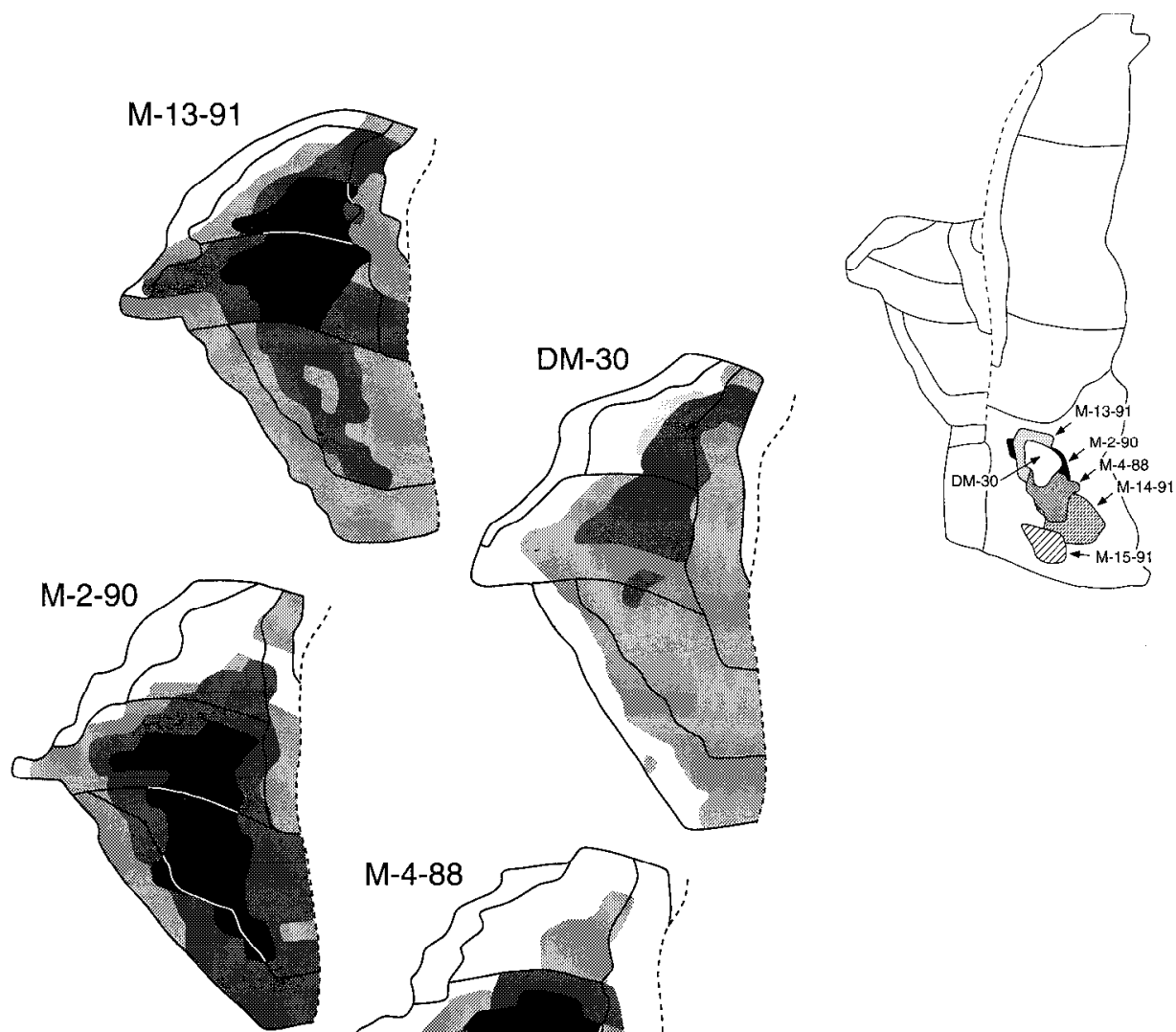

Figure 9. A series of two-dimensional unfolded maps of the entorhinal cortex illustrating the distribution and density of terminal labeling after six different ${ }^{3} \mathrm{H}$-amino acid injections in the parahippocampal cortex. At the top right is an unfolded map showing the location of the anterograde injections within the parahippocampal cortex (Fig. 2A). Black areas represent the region where the heaviest terminal labeling was observed, the two progressively lighter shades of gray indicate the regions where progressively lighter terminal labeling was observed. White areas represent regions where no terminal labeling was observed. See Materials and Methods for more details on the construction of these density maps. Solid lines represent the boundaries of the different subdivisions of the entorhinal cortex. Broken line represents the rhinal sulcus. Scale bar, $2 \mathrm{~mm}$.
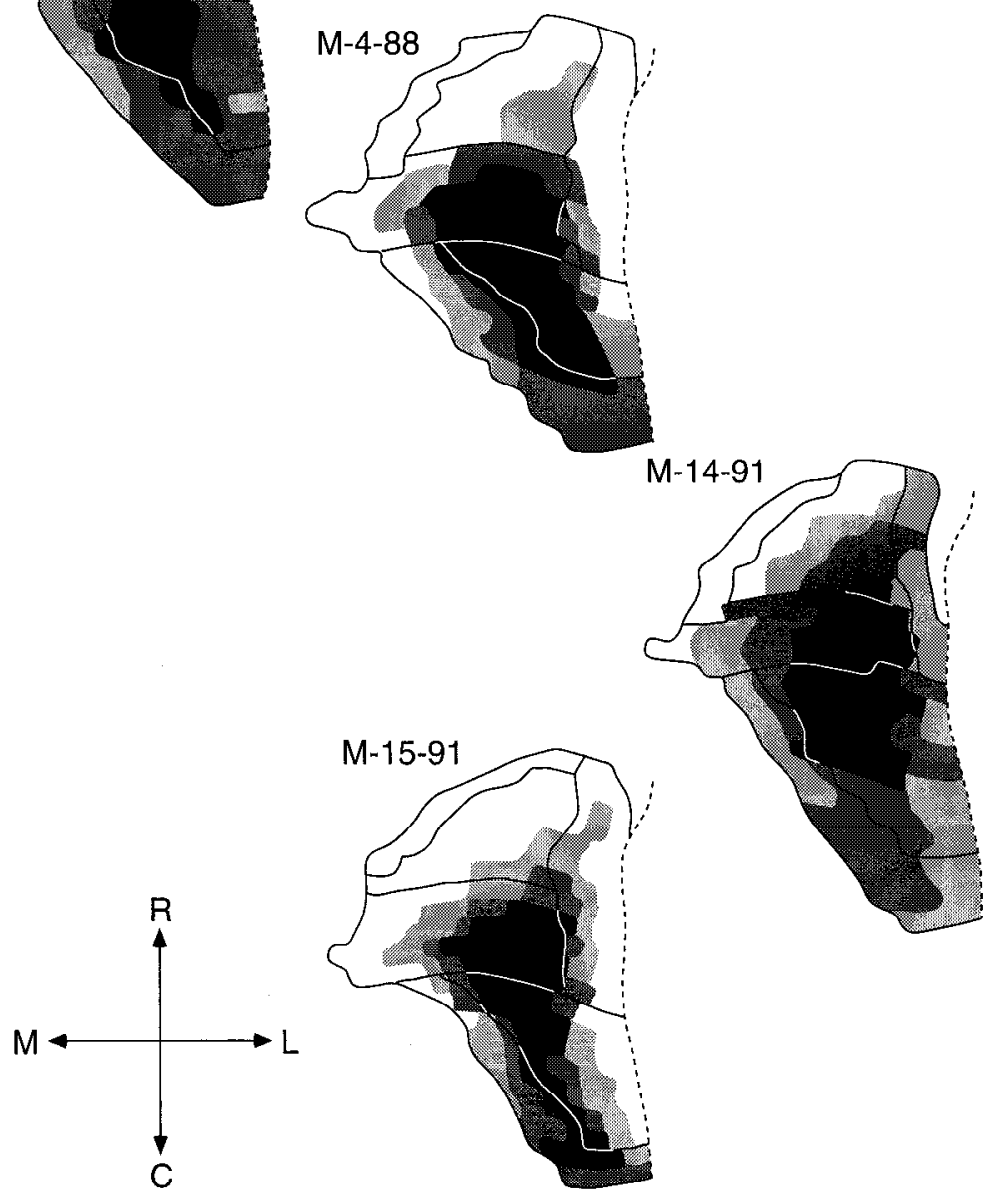

Figure 10. A series of two-dimensional unfolded maps of the entorhinal cortex illustrating the relative density of labeled cells in 10 different cases with retrograde tracer injections in the parahippocampal cortex. At the top right is an unfolded map showing the relative location of the retrograde tracer injections in the parahippocampal cortex. Black voxels represent areas with the densest retrograde labeling and progressively lighter shades of gray represent progressively lower density of labeling. See Materials and Methods for more details on the construction of these density maps. Solid lines represent the boundaries of the different subdivisions of the entorhinal cortex. Dashed line represents the rhinal sulcus. Scale bar, 2 mm. 


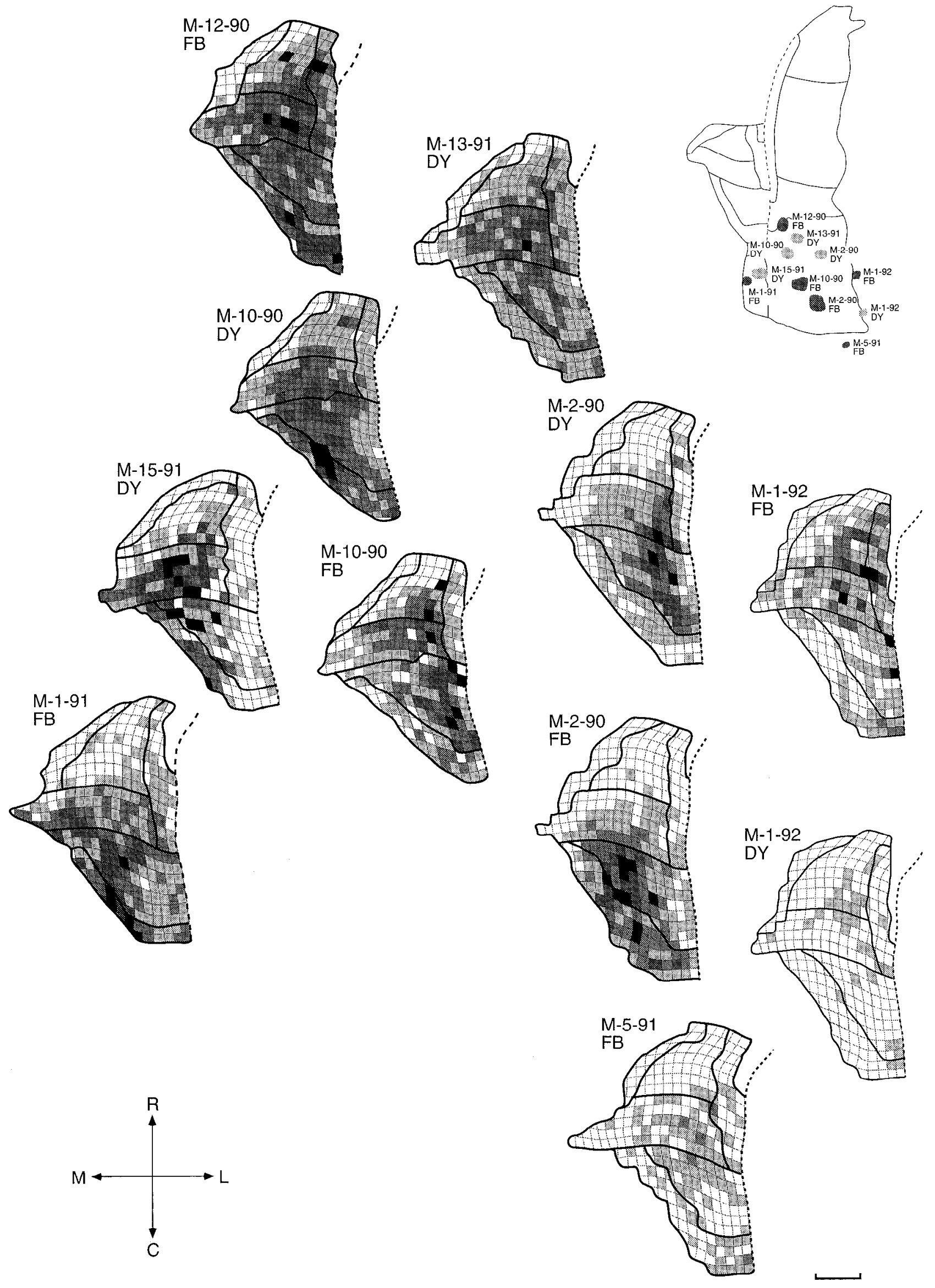


in and around layer II, including the superficial part of layer III and the deep portions of layer I. In most cases, relatively strong terminal labeling was also observed in layers V and VI (Fig. 8ac).

Organization of the reciprocal projections between the entorhinal cortex and the parahippocampal cortex

Projections from the parahippocampal cortex to the entorhinal cortex

Anterograde tracer experiments. The locations of six representative injections of ${ }^{3} \mathrm{H}$-amino acids in area $\mathrm{TF}$ of the parahippocampal cortex are shown in Figure $2 B$. While this series of injections spanned the rostrocaudal extent of area $T F$, all six were located at approximately the same mediolateral position. The patterns of terminal labeling in the entorhinal cortex resulting from these injections are illustrated in a series of twodimensional unfolded maps in Figure 9.

In all cases, injections in area TF of the parahippocampal cortex resulted in substantial fiber and terminal labeling that was distributed throughout approximately the caudal two-thirds of the entorhinal cortex. Terminal labeling was consistently highest at mid-mediolateral portions of the caudal two-thirds of the entorhinal cortex. The more rostral injections (such as experiment M-13-91) tended to project more heavily to the rostral portion of the longitudinally oriented band while the more caudally placed injections (such as M-15-91) projected most strongly to caudal levels of the longitudinally oriented band. Since more medial or more lateral injections within the parahippocampal cortex were not available for analysis, it can not be determined whether injections in these regions would give rise to more laterally or medially situated bands of highest terminal labeling in the entorhinal cortex. We will comment on this further in the next section when discussing the distribution of labeled cells in the parahippocampal cortex following retrograde tracer injections in the entorhinal cortex.

Retrograde tracer injections. As noted in the section on the perirhinal cortex, we have reevaluated the retrograde data of Insausti et al. (1987) to seek confirmation of the topography of parahippocampal projections to the entorhinal cortex derived from the anterograde tracer studies described above (Fig. $2 B$ illustrates the entorhinal injection sites of the retrograde tracers, and Table 1 shows the percentages of labeled cells in the perirhinal and parahippocampal cortices resulting from these injections).

As expected from the results of the anterograde tracer experiments in area $\mathrm{TF}$, the largest percentage of retrogradely labeled cells in the parahippocampal cortex was observed after injections involving the caudal portion of the entorhinal cortex (cases M-4-86, IM-3, IM-1, IM-7, DM-45, and IM-10). Conversely, injections involving the rostral portion of the entorhinal cortex resulted in relatively low numbers of labeled cells (IM-6 and IM-8) or no labeled cells (IM-4) in the parahippocampal cortex. The data from the retrograde tracer experiments in the entorhinal cortex also provide additional information concerning the mediolateral topography of projections from the parahippocampal cortex to the entorhinal cortex. As noted above, the library of cases of ${ }^{3} \mathrm{H}$-amino acid injections only involved the midmediolateral extent of TF and no injections were placed in TH. However, in the retrograde cases it was clear that the injections involving the caudal medial aspect of the entorhinal cortex (cases M-4-86, IM-3, IM-1, and IM-10) produced the largest percentage of retrogradely labeled cells in area $\mathrm{TH}$ of the parahip- pocampal cortex whereas more laterally situated injections (cases IM-7 and DM-45) led to relatively little labeling in area $\mathrm{TH}$. This mediolateral topography can be appreciated by examining Figures 12 and 13 in Insausti et al. (1987). Figure 13 of that article illustrated the distribution of retrogradely labeled cells in case M-4-86, which contained an injection in the medial portion of the entorhinal cortex. This case exhibited the largest numbers of retrogradely labeled cells in area $\mathrm{TH}$ and medial area TF (their Fig. 13H,I). Figure 12 in Insausti et al. (1987) illustrated the distribution of retrogradely labeled cells in case IM-7, which received a retrograde tracer injection in the lateral portion of the entorhinal cortex. This case exhibited many fewer labeled cells in area TH, and the labeled cells in area TF appeared to be shifted laterally compared to the labeling in case M-4-86 (their Fig. $12 N-P$ ). More recently analyzed cases containing restricted injections in the lateral portions of the entorhinal cortex (cases M-1-90 FB, M-11-90 DY, and M-11-90 FB; Fig. $2 B$ ) largely confirm the pattern of labeling seen in case IM7. Anterograde tracer injections in area $\mathrm{TH}$ and the lateral extreme of area TF will be needed to confirm this mediolateral topography and to determine the strength of these projections.

To summarize, data from both the anterograde and retrograde experiments suggest that the parahippocampal cortex projection to the entorhinal cortex follows a mediolateral topography (see Fig. $12 C$ ) with a less prominent rostrocaudal component. In general, the parahippocampal cortex projects most heavily to approximately the caudal two-thirds of the entorhinal cortex. Rostral portions of parahippocampal cortex tend to project more strongly to the rostral levels of the entorhinal cortex while caudal portions of the parahippocampal cortex project to the caudal entorhinal cortex. There also appears to be a mediolateral topography such that medial portions of the parahippocampal cortex (area TH and medial area TF) project medially in the entorhinal cortex while lateral portions of the parahippocampal cortex (lateral area TF) project to more lateral portions of the entorhinal cortex.

\section{Projections from the entorhinal cortex to the parahippocampal cortex}

Retrograde tracer experiments. Unlike the ${ }^{3} \mathrm{H}$-amino acid injections in the parahippocampal cortex, which had a relatively limited mediolateral distribution, the retrograde tracer injections involved all portions of the parahippocampal cortex. The sizes and locations of 11 representative retrograde tracer injections in and around the parahippocampal cortex are illustrated in Figure $2 B$. The relative density and distribution of retrogradely labeled cells in the entorhinal cortex resulting from these injections are summarized in the series of two-dimensional unfolded maps shown in Figure 10. In general, the largest number of retrogradely labeled cells was observed in the caudal twothirds of the entorhinal cortex. Moreover, as with the projection from the parahippocampal cortex to the entorhinal cortex, the projection from the entorhinal back to the parahippocampal cortex exhibited both a mediolateral and a weak rostrocaudal topographic organization. Three of these cases were located either at the lateral border (M-1-92 DY, M-1-92 FB) or just caudal (M-5-91 FB) to the apparent cytoarchitectonic boundary of the parahippocampal cortex. The organization of the labeling in these cases will also be considered.

The mediolateral aspect of the organization of this projection can be appreciated by comparison of cases M-15-91 DY, M-10- 
$90 \mathrm{FB}$, and M-1-92 FB. The retrograde tracer injections in these cases were located at approximately the same rostrocaudal level of the parahippocampal cortex but varied in their mediolateral position. The injection in case M-15-91 DY was focused in the lateral portion of caudal area $\mathrm{TH}$. The highest density of retrogradely labeled cells was observed in the medial portion of the caudal entorhinal cortex (Fig. 10). In contrast, case M-10$90 \mathrm{FB}$ contained a retrograde tracer injections situated laterally in area TF, and the highest density of retrogradely labeled cells in this case was located laterally in the caudal entorhinal cortex. The injection in case M-1-92 FB was located just lateral to the cytoarchitectonic border of area TF, yet the overall pattern of labeling was still observable in this case. This pattern of labeling may suggest that the transitional areas between area TF and area TE can exhibit patterns of connectivity that are characteristic of lateral portions of area TF

The same mediolateral pattern can be seen by comparing case M-10-90 DY with case M-2-90 DY. The injection in experiment M-10-90 DY was located medially in area TF and produced the highest density of labeling medially in the entorhinal cortex whereas the injection in case M-2-90 DY was located more laterally in TF and produced the highest density of labeled cells more laterally in the entorhinal cortex.

Special cases to consider are case M-1-92 DY, M-1-92 FB, and M-5-91 FB. Cases M-1-92 FB and M-1-92 DY contained injections located at the lateral extreme of area TF at a mid and caudal level of the parahippocampal cortex, respectively. While both cases followed the same overall mediolateral topography described above, case M-1-92 FB exhibited a density of retrogradely labeled cells in the entorhinal cortex that was within the range of other cases, while case M-1-92 DY exhibited very few labeled cells in the entorhinal cortex (shaded voxels represent between one and three labeled cells). Case M-5-91 FB contained an injection site that was apparently situated just caudal to the caudal border of the parahippocampal cortex. This case also produced very few retrogradely labeled cells in the entorhinal cortex (lightest shaded voxels contained between one and four labeled cells, and voxels represented in the next darkest shading pattern contained between five and seven labeled cells); however, the overall pattern of labeling was consistent with the mediolateral and rostrocaudal topography discussed above. The strikingly low number of labeled cells in the entorhinal cortex in cases M-1-92 DY and M-5-91 FB suggests there can be a rapid drop in the density of connections with the entorhinal cortex as one moves from the parahippocampal cortex into adjacent cortical areas. The higher density of labeling observed in case M-1-92 FB, however, suggests that there may be some variability in the actual transition between area TF and laterally adjacent cortex relative to the apparent cytoarchitectonic boundary. Taken together, these cases suggest that the zone of transition between one area and the next may be more gradual and slightly more variable than portrayed by the solid boundary lines typically shown between adjacent cortical areas.

There appeared to be a subtle rostrocaudal component of the topography in the projection from the entorhinal cortex to the parahippocampal cortex. Compare cases M-13-91 DY and M-10$90 \mathrm{DY}$, which contained injections in the rostral part of the parahippocampal cortex, with case M-2-90 FB, which contained an injection in the caudal portion of the parahippocampal cortex. Whereas both cases with rostrally situated injections demonstrated some labeled cells in the rostral portion of the entorhinal cortex, the case with a caudal parahippocampal cortex
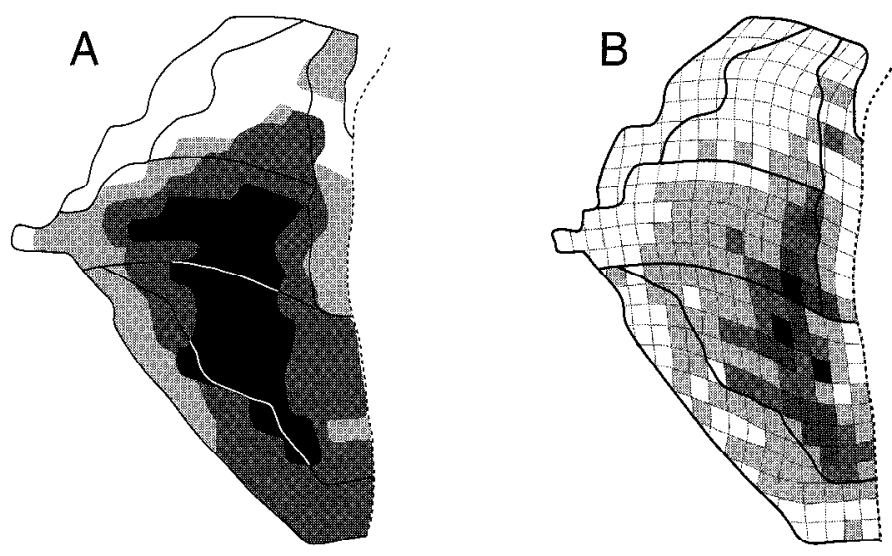

Figure 11. Unfolded two-dimensional maps of case M-2-90. A, Unfolded map illustrates the distribution of terminal labeling in the entorhinal cortex after the ${ }^{3} \mathrm{H}$-amino acid injection in this case (see Fig. $2 A$ ). $B$, Unfolded map illustrates the distribution and density of retrogradely labeled cells in the same case after an injection of DY.

injection demonstrated few, if any, cells in the rostral portion of the entorhinal cortex.

The organization of projections from the entorhinal cortex to the parahippocampal cortex can be summarized as follows (see Fig. 12D). In general, the caudal two-thirds of the entorhinal cortex provides the strongest projection to the parahippocampal cortex. Cells in the medial aspect of the caudal entorhinal cortex project to the medial aspects of the parahippocampal cortex (area TH and medial area TF) while cells in the lateral aspects of the caudal entorhinal cortex tend to project to the lateral portion of the parahippocampal cortex. There is also a small but detectible rostrocaudal component to the topography; cells in rostral portions of the entorhinal cortex tend to project only to the rostral parahippocampal cortex whereas cells in the caudal entorhinal cortex project strongly to much of the rostrocaudal extent of the parahippocampal cortex.

Anterograde tracer experiments. In order to confirm the topography of projections from the entorhinal cortex to the parahippocampal cortex, the distribution of labeled fibers and terminals in the parahippocampal cortex resulting from anterograde tracer injections in the entorhinal cortex was examined. Three representative cases are shown in Figure 5.

The injection in case DM-13 involved the lateral aspect of the caudal entorhinal cortex. As expected from the findings of the retrograde tracer studies, the strongest terminal labeling in the parahippocampal cortex was observed in the mid to lateral aspects of area TF while area TH contained no terminal labeling. A similar pattern of labeling was observed in case M-10-87, which contained an anterograde tracer injection located more rostrally in arca $\mathrm{E}_{\mathrm{L}}$ of the entorhinal cortex.

In contrast to these cases, the injection in case DM-25, which involved the medial aspect of the rostral entorhinal cortex, produced the strongest labeling medially in the parahippocampal cortex at the TH/TF border. Because this injection was located fairly far rostrally, the overall labeling in the parahippocampal cortex tended to be weak.

Thus, anterograde tracer injections in the entorhinal cortex confirmed the mediolateral organization of the projections from the entorhinal cortex to the parahippocampal cortex. The medial portions of the entorhinal cortex tend to project more strongly to the medial aspect of the parahippocampal cortex, and the 


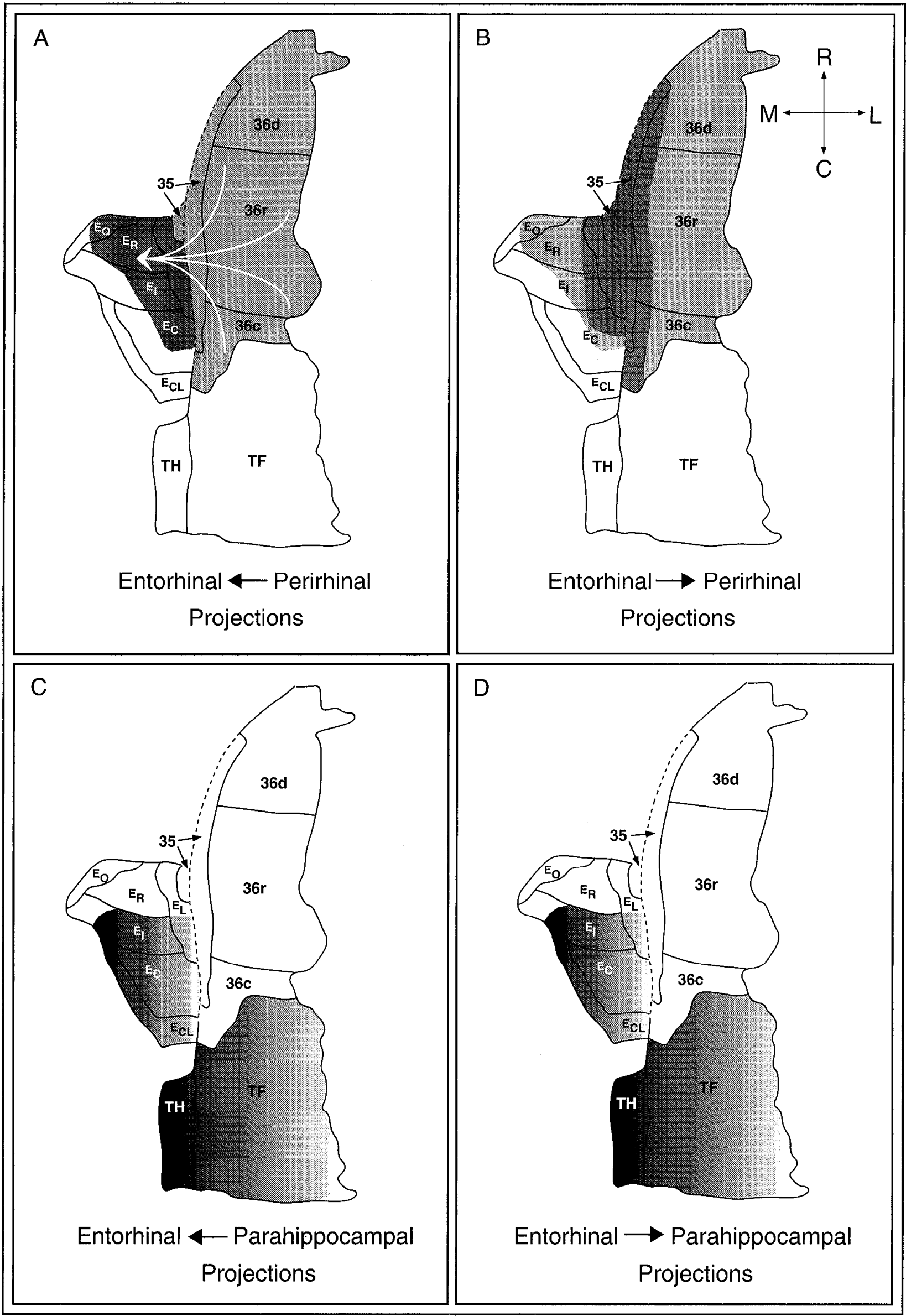


lateral regions of the entorhinal cortex project most heavily to the lateral aspect of the parahippocampal cortex.

\section{The degree of reciprocity of projections of the entorhinal cortex with the parahippocampal cortex}

By comparing the unfolded maps in Figures 9 and 10, it is clear that interconnections between the entorhinal cortex and the parahippocampal cortex appear to have a similar topography and are therefore likely to be tightly reciprocal. We were able to evaluate the extent of reciprocity more directly by studying experiment M-2-90 in which an anterograde tracer injection was located approximately $1.77 \mathrm{~mm}$ medial to a retrograde tracer injection at the same rostrocaudal level of area TF of the parahippocampal cortex. Although the cores of both the anterograde and retrograde injection sites were located in the parahippocampal cortex, there was some minor involvement of the overlying CA 1/subicular border region by the anterograde tracer injection. Figure 11 shows two replicas of an unfolded map of the entorhinal cortex in case M-2-90, one showing the pattern of anterograde labeling (Fig. 11A) and the other showing the density of retrogradely labeled cells (Fig. $11 B$ ). Both injections produced a heavy band of either anterograde or retrograde labeling through approximately the caudal two-thirds of the entorhinal cortex. However, this longitudinally organized band of labeling was shifted slightly laterally in the entorhinal cortex in the retrograde case compared to the anterograde case. We attribute this shift in highest density of labeling not to a different topographic organization of the reciprocal projections but rather to the fact that the retrograde tracer injection in area TF was located just lateral to the lateral boundary of the anterograde tracer injection (compare case M-2-90 in Fig. $2 A$ to case M-2$90 \mathrm{DY}$ in Fig. $2 B$ ). As predicted by the topography of the entorhinal/parahippocampal connections, the retrograde labeling was shifted laterally in the entorhinal cortex compared to the anterograde labeling. We would predict, therefore, that if the anterograde and retrograde tracers were injected at the same site in the parahippocampal cortex, the labeling from the two tracers would have been essentially superimposable.

The highly reciprocal pattern of entorhinal-parahippocampal connections can also be appreciated by examining case M-1391. In this case, the center of the anterograde tracer injection was located approximately $1.9 \mathrm{~mm}$ more caudal to the center of the retrograde tracer injection in the rostral portion of area TF (Fig. 2). The distribution of terminal labeling in case M-1391 (Fig. 9) is very similar to the distribution of retrogradely labeled cells in case M-13-91 DY (Fig. 10). Taken together, these data suggest that the connections between the parahip- pocampal cortex and the entorhinal cortex demonstrate a high degree of reciprocity.

Laminar organization of the reciprocal projections between the entorhinal cortex and the parahippocampal cortex

Projections from the parahippocampal cortex to the entorhinal cortex

Cells of origin. The projection to the entorhinal cortex from the parahippocampal cortex arises mainly in layer III with a smaller contribution from layer V (Insausti et al., 1987).

Laminar organization of terminations. Figure $7 B$ shows the pattern of termination in a series of representative coronal sections through the entorhinal cortex after an anterograde tracer injection in area TF (case M-13-91). Following anterograde tracer injections in the parahippocampal cortex, the strongest labeling was generally observed in layers I, II, and III of the entorhinal cortex. However, in areas where the labeling was strongest, there was moderate to heavy terminal labeling in deeper layers as well.

\section{Projections from the entorhinal cortex to the parahippocampal cortex}

Cells of origin. The vast majority of retrogradely labeled cells in the entorhinal cortex after injections in the parahippocampal cortex were observed in layer $\mathrm{V}$, with fewer labeled cells in layer VI and only occasional cells in layer III.

Laminar organization of termination. After anterograde tracer injections in the entorhinal cortex, the strong terminal labeling in areas TH and TF was consistently observed in layers I, II, and superficial III, as well as in deep layers V and VI (Fig. $8 c, d$ ).

\section{Discussion}

Studies in humans (Scoville and Milner, 1957), monkeys (Mishkin, 1978), and rats (Morris et al., 1982) have provided convincing evidence that the hippocampal formation is intimately involved in memory function. Multidisciplinary attempts to determine the full scope of this memory system have demonstrated that cortical areas adjacent to the hippocampal formation, including the perirhinal and parahippocampal cortices, also contribute substantively to normal memory function (ZolaMorgan et al., 1989b; Meunier et al., 1990; Suzuki et al., 1993). While the entorhinal cortex is the major route through which cortical information enters the rest of the hippocampal formation, the perirhinal and parahippocampal cortices are the major source of cortical input to the entorhinal cortex. Thus, the hippocampal formation, and perirhinal and parahippocam-

Figure 12. Schematic representation of the organization of the reciprocal connections of the entorhinal cortex with the perirhinal and parahippocampal cortices. $A$, Organization of the projections from the perirhinal cortex to the entorhinal cortex. All areas of perirhinal cortex (shown in light gray) project to the same regions of the rostral and lateral portions of the entorhinal cortex (indicated in dark gray). B, Organization of the projections from the entorhinal cortex to the perirhinal cortex. The medial portion of the entorhinal cortex (shown in light gray) projects to lateral regions of the perirhinal cortex (also shown in light gray) while lateral portions of the entorhinal cortex (shown in dark gray) project to medial areas of the perirhinal cortex (also shown in dark gray). $C$, Organization of the projections from the parahippocampal cortex to the entorhinal cortex. Medial portions of the parahippocampal cortex (shown in the darkest shades of gray) project to the medial entorhinal cortex (also shown in the darkest shades of gray) while progressively more lateral portions of the parahippocampal cortex (shown in progressively lighter shades of gray) project to progressively more lateral portions of the entorhinal cortex (also shown in lighter shades of gray). D, Organization of the projections from the entorhinal cortex to the parahippocampal cortex. Like the projections from the parahippocampal cortex to the entorhinal cortex, the reciprocal projections also exhibit a strong mediolateral topography. Thus, medial portions of the caudal entorhinal cortex (shown in the darkest shades of gray) project to medial portions of the parahippocampal cortex (also shown in the darkest shades of gray) and progressively more lateral portions of the caudal entorhinal cortex (shown in progressively lighter shades of gray) project to progressively more lateral portions of the parahippocampal cortex (also shown in progressively lighter shades of gray). 
pal cortices together appear to constitute a multicomponent medial temporal lobe memory system.

In the present study we have attempted to determine the topographic and laminar organization of the reciprocal connections of the monkey entorhinal cortex with the perirhinal and parahippocampal cortices. This study comes in the context of a broader program of research designed to examine the cytoarchitectonic and connectional characteristics of the perirhinal and parahippocampal cortices in the monkey. There were three major findings in the present studies. First, the topographic organization of entorhinal/perirhinal interconnections was distinct from entorhinal/parahippocampal connections. Second, the degree of reciprocity of these interconnections, that is, the extent of point-to-point or region-to-region mapping of inputs and outputs, was also different for the perirhinal and parahippocampal cortices. Third, the perirhinal and parahippocampal projections to the entorhinal cortex resemble a feedforward pattern while the entorhinal projections to the perirhinal and parahippocampal cortices resemble feedback projections.

\section{Differences in the topographic organization of perirhinal and parahippocampal connections with the entorhinal cortex}

The organization of the projections from the perirhinal and parahippocampal cortices to the entorhinal cortex is summarized in Figure 12. The projections from the perirhinal and parahippocampal cortices terminated in different regions of the entorhinal cortex and also differed in the extent of convergence of their terminal fields within the entorhinal cortex. The perirhinal cortex tended to project most strongly to the rostral twothirds of the entorhinal cortex while the parahippocampal cortex projected most strongly to the caudal two-thirds. One of the most striking findings of this study was that all regions of the perirhinal cortex projected in a highly convergent manner onto the rostral and lateral portions of the entorhinal cortex (Fig. $12 A$ ). In contrast, the projections from the parahippocampal cortex to the entorhinal cortex exhibited a topographic organization; the medial regions of the parahippocampal cortex tended to project to the caudomedial portion of the entorhinal cortex while lateral portions of the parahippocampal cortex tended to project to the caudolateral portion of the entorhinal cortex (Fig. 12C). Projections from any position in the perirhinal or parahippocampal cortex tended to be quite divergent; that is, they extended throughout a large rostrocaudal and mediolateral area of the entorhinal cortex. Thus, although projections from the perirhinal and parahippocampal cortices appeared to be organized differently, there was, nonetheless, substantial overlap of the terminal fields of these areas, particularly in the mid-portion of the lateral division of the entorhinal cortex (Fig. 12).

Our finding of particularly heavy innervation of the midportion of the lateral entorhinal cortex from both the perirhinal and parahippocampal cortices is consonant with earlier studies in both the monkey and the cat that emphasized the importance of laterally situated portions of the entorhinal cortex as a zone of convergence for cortical inputs (Van Hoesen and Pandya, 1975a: Room and Groenewegen, 1986).

The finding that the perirhinal and parahippocampal cortices appear to have distinct patterns of interconnections with the entorhinal cortex is consistent with a growing body of evidence indicating that the perirhinal and parahippocampal cortices themselves receive distinct complements of cortical afferents (Suzuki and Amaral, 1990). The perirhinal cortex receives inputs from unimodal visual areas in the temporal lobe (Suzuki and Amaral, 1990; Webster et al., 1991; Martin-Elkins and Horel, 1992) as well as presumed polysensory information from areas including the parahippocampal cortex and cingulate cortex (Suzuki and Amaral, 1990). These anatomical findings have been confirmed by electrophysiological studies in the monkey which found that cells in the perirhinal cortex respond to polysensory stimuli (Desimone and Gross, 1979). However, more recent studies have focused on the strong visual and memoryrelated responsivity of cells in and around the perirhinal area (Miyashita and Chang, 1988; Miller et al., 1991, 1993; Riches et al., 1991).

In contrast to the heavily unimodal sensory influence on perirhinal cortex, the parahippocampal cortex receives strong inputs from polymodal areas located, for example, in the dorsal bank of the superior temporal sulcus (Suzuki and Amaral, 1990) and in the retrosplenial cortex (Suzuki and Amaral, 1990), visuospatial information from the posterior parietal lobe (Jones and Powell, 1970; Seltzer and Pandya, 1976; Andersen et al., 1990), as well as unimodal auditory information from area TPT of the superior temporal gyrus (Tranel et al.; 1988). Compared to the perirhinal cortex, the parahippocampal cortex receives relatively little direct input from the unimodal visual area TE, although it does receive unimodal visual input from areas VTF and V4. Electrophysiological studies in the monkey have confirmed that the cells of the parahippocampal cortex respond to stimuli in two or more sensory modalities (Desimone and Gross, 1979).

Given the differences between the perirhinal and parahippocampal cortices in both their cortical inputs and their interconnections with the entorhinal cortex, it is of interest to consider how the topographical differences in the projections of these regions to the entorhinal cortex might relate to the organization of the perforant path projections to the dentate gyrus and other subareas of the hippocampal formation. In the cat (Witter and Groenewegen, 1984) and monkey (Witter et al., 1989), the laterally situated portions of the entorhinal cortex project preferentially to caudal (septal in the rat and cat) levels of the dentate gyrus and hippocampus while progressively more medially situated portions of the entorhinal cortex project to progressively more rostral (temporal) levels of the dentate gyrus and hippocampus. A rostrocaudal component of the organization of the perforant path projection determines the radial distribution of terminal labeling in the dentate gyrus; rostral levels of the entorhinal cortex project to more superficial portions of the molecular layer of the dentate gyrus while progressively more caudal levels of the entorhinal cortex terminate in progressively deeper portions of the molecular layer (Witter and Amaral, 1991). Different rostrocaudal levels of the entorhinal cortex also project differentially to CA1 and the subiculum (Witter and Amaral, 1991). In this case, the projections go to different transverse portions of the fields. Thus, rostral levels of the entorhinal cortex project to the CAl/subiculum border region whereas progressively more caudal portions of the entorhinal cortex project either more proximally in CAl or more distally in the subiculum.

Do the projection patterns of the perforant path provide any insight into which regions of the dentate gyrus, hippocampus, and subiculum might be more influenced by information arriving via the perirhinal versus parahippocampal cortices? Although the extensive divergence of the projections from the perirhinal and parahippocampal cortices to the entorhinal cortex precludes the possibility of a precise point-to-point mapping, there are at least two general trends that can be appreciated. 
First, because the projections from both the perirhinal and parahippocampal cortices involve much of the mediolateral extent of the entorhinal cortex, projections from these fields will influence much of the rostrocaudal extent of the dentate gyrus and hippocampus. However, because the parahippocampal cortex tends to project farther medially in the entorhinal cortex than the perirhinal cortex, the parahippocampal cortex may have somewhat more influence on the most rostral levels of the dentate gyrus. Second, since projections from perirhinal and parahippocampal cortices terminate at different rostrocaudal levels of the entorhinal cortex, there is a potential difference in the radial position of the dentate gyrus molecular laycr that may receive perirhinal versus parahippocampal input and a different transverse distribution of input within CA1 and the subiculum. The projections from the perirhinal cortex, arriving in the rostral two-thirds of the entorhinal cortex, would tend to be relayed by fibers of the perforant path that terminate at a more superficial level of the dentate molecular layer and closer to the CAl/ subiculum border. Inputs from the parahippocampal cortex, arriving at the caudal portion of the entorhinal cortex, are relayed by perforant path fibers that terminate at deeper levels of the dentate molecular layer, more proximal portions of CAl, and more distal portions of subiculum. Another possible factor differentiating the perirhinal and parahippocampal input to the entorhinal cortex is suggested by electrophysiological studies in the rat. There are two distinct perforant pathways in the rat, the lateral and medial perforant pathways, which not only have distinct topographical organizations, but also have distinct clectrophysiological properties (McNaughton, 1980). The cells of origin of the lateral and medial perforant pathways in the rat correspond roughly to the rostral and caudal entorhinal cortex, respectively, in the monkey. Thus, it will be of substantial interest to determine if projections arising from different rostrocaudal levels of the monkey entorhinal cortex also demonstrate distinct electrophysiological attributes and if these electrophysiological differences may underlie differences in processing perirhinal versus parahippocampal input.

An additional anatomical differentiation between the perirhinal and parahippocampal inputs that may have some functional relevance is the degree of convergence of the two projections. As described above, while the perirhinal cortex exhibits extensive convergence within the entorhinal cortex, the parahippocampal projections are more topographically organized. Although it is unclear at this time what the degree of convergence means physiologically, it is tempting to speculate that this anatomical difference could underlie differences in how primarily visual information arriving via the perirhinal cortex versus strongly polymodal information arriving via the parahippocampal cortex may be processed within the hippocampal formation.

The origins of the entorhinal projections to the perirhinal and parahippocampal cortices roughly paralleled the topography of the inputs from these regions. In general, layer $\mathrm{V}$ cells of the caudal portion of the entorhinal cortex projected preferentially to the parahippocampal cortex whereas the rostral portion of the entorhinal cortex projected more heavily to the perirhinal cortex. The entorhinal projections demonstrated substantial convergence and divergence. Cells in any particular area of the entorhinal cortex projected to much of the perirhinal or parahippocampal cortices, and thus any particular point in the perirhinal and parahippocampal cortex received convergent input from a large area of the entorhinal cortex.

The rough rostrocaudal organization of the connections between the entorhinal cortex and the perirhinal and parahippo- campal cortices is paralleled by other afferent and efferent connections of the entorhinal cortex. The lateral nucleus of the amygdala, for example, projects to layer III of the entorhinal cortex, but only to the rostral division (Saunders and Rosene, 1988; A. Pitkänen and D. G. Amaral, unpublished observations). A complementary projection originates from the presubiculum that also terminates primarily in layer III, but of the caudal portion of the entorhinal cortex (fields $\mathrm{E}_{\mathrm{I}}, \mathrm{E}_{\mathrm{C}}$ and $\mathrm{E}_{\mathrm{CL}}$ ) (D. G. Amaral and M. P. Witter, unpublished observations).

\section{How tight is the reciprocity of entorhinal/perirhinal and entorhinal/parahippocampal connections?}

It has been known for some time that corticocortical projections within the visual and somatosensory systems tend to be reciprocal (Kuypers et al., 1965; Jones, 1967; Tigges et al., 1973; Rockland and Pandya, 1979). Moreover, in the primary visual (Tigges et al., 1973) and somatosensory areas (Jones, 1967), the point-to-point reciprocity of these areas with secondary sensory areas has been well documented. The degree of reciprocity of higher-order cortical association areas, however, has rarely been directly evaluated (see Felleman and Van Essen, 1991).

We have shown that the degree of reciprocity of the interconnections between the perirhinal and entorhinal cortices appears to be different from that between the parahippocampal and entorhinal cortices (Fig. 12). The parahippocampal cortex is interconnected with the entorhinal cortex with a high degree of reciprocity (i.e., the same region of the entorhinal cortex that receives a strong input from the parahippocampal cortex also returns a strong projection back) whereas the degree of reciprocity of the perirhinal cortex with the entorhinal cortex varies depending on the mediolateral part of the perirhinal cortex; medial portions of the perirhinal cortex tend to have a higher degree of reciprocity with the entorhinal cortex than lateral portions. The functional implications of these variations in the extent of reciprocity may become more apparent as additional neuroanatomical and electrophysiological information is obtained concerning the intrinsic organization of the monkey entorhinal cortex.

\section{Are the perirhinal and parahippocampal projections to the entorhinal cortex of the feedforward or feedback type?}

It is of interest to compare the laminar organization of connections of the entorhinal cortex with the perirhinal and parahippocampal cortices with the organization of other corticocortical connections. Corticocortical projections tend to follow particular patterns of origin and termination. Rockland and Pandya (1979) characterized these patterns in the connections of primary visual and visual association cortices. More recently, Felleman and Van Essen (1991) have divided all known visual corticocortical connections into three different categories based on layers of origin and termination: ascending or feedforward projections, lateral projections, and descending, or feedback projections. Despite modifications necessitated by the periallocortical cytoarchitecture of the entorhinal cortex, the projections from the perirhinal and parahippocampal cortices to the entorhinal cortex appear to exhibit a feedforward type of organization. As in other feedforward projections, the projections from the perirhinal and parahippocampal cortices originate primarily from superficial layer III and from layer V. Unlike other feedforward projections that terminate mainly in layer IV, however, the perirhinal and parahippocampal fibers terminate primarily in layers II and III of the entorhinal cortex. Since the entorhinal cortex does not have a typical layer IV (internal 
granular layer), it is not surprising that more superficially placed cells are in receipt of the feedforward projection. Moreover, these projections are feedforward in the sense that they terminate on the superficial cells of the entorhinal cortex that then project forward to the dentate gyrus and hippocampus. The projection from the entorhinal cortex to the perirhinal and parahippocampal cortices follows a classic feedback pattern; the projection originates in layer $\mathrm{V}$ and terminates mainly in layer I and to a lesser extent in layer $\mathrm{V}$.

\section{Functional implications}

The results of the studies reported in this article demonstrate a strong neuroanatomical relationship between the perirhinal and parahippocampal cortices, on the one hand, and the entorhinal cortex on the other. The entorhinal cortex is widely viewed as the gateway by which sensory information attains the other fields of the hippocampal formation, and thus the perirhinal and parahippocampal cortices have been considered to be the major sources of this information. Historically, the focus of attention for medial temporal lobe memory function has been the hippocampal formation while the perirhinal and parahippocampal cortices have typically been portrayed as "relays" or "interfaces" for communication between the hippocampal formation and the neocortex. However, if the perirhinal and parahippocampal cortices were acting simply as relays of information to the hippocampal formation and entorhinal cortex, one would be forced to predict that damage to the entorhinal cortex would produce a memory impairment that was as severe as, or more severe than, that caused by damage to the perirhinal and parahippocampal cortices. However, recent data from two laboratories indicate that this is not the case.

Bilateral lesions of the monkey perirhinal and parahippocampal cortices, which spare the entorhinal cortex and other fields of the hippocampal formation, produce a robust and enduring memory deficit on a widely used task of visual recognition memory in the monkey, the delayed nonmatching to sample task (Zola-Morgan et al., 1989b; Suzuki et al., 1993). In contrast, animals with bilateral lesions confined to the entorhinal cortex exhibit a significant but markedly less severe memory impairment on this task (Leonard et al., 1992).

These results require several modifications of the historically held view concerning the relative roles of medial temporal lobe structures in memory function. First, these results support the idea that the perirhinal and parahippocampal cortices contribute directly to medial temporal lobe memory function. Moreover, because the deficit associated with lesions of the perirhinal and parahippocampal cortices is substantially greater than that associated with entorhinal lesions, it appears that the perirhinal and parahippocampal cortices may be even more important than the entorhinal cortex in mediating the memory functions required for the performance of the delayed nonmatching to sample task.

Although it is clear from buth human (Zold-Morgan et al., 1986; Victor and Agamanolis, 1990) and rat (Morris et al., 1982; Rudy and Sutherland, 1989) studies that the hippocampal formation and entorhinal cortex contribute significantly to memory function, the question remains of how we can better define the role of these structures in a nonhuman primate model of human amnesia. A profitable perspective may be in considering that the entorhinal cortex may use the convergence of all of its cortical inputs, including those from the perirhinal and parahippocampal cortices, to carry out computations or processes that are distinct from those carried out by either the perirhinal or parahippocampal cortex. If so, it appears that a behavioral task that engages the precise memory functions of the entorhinal cortex remains to be developed.

\section{References}

Amaral DG, Price JL (1983) An air pressure system for the injection of tracer substances into the brain. J Neurosci Methods 9:35-43.

Amaral DG, Insausti R, Cowan WM (1987) The entorhinal cortex of the monkey. I. Cytoarchitectonic organization. J Comp Neurol 264: 326-355

Andersen RA, Asanuma C, Essick G, Siegel RM (1990) Corticocortical connections of anatomically and physiologically defined subdivisions within the inferior parietal lobule. J Comp Neurol 296:65-113.

Cowan WM, Gottlieb DI, Henrickson AE, Price JL, Woolsey TA (1972) The autoradiographic demonstration of axonal connections in the central nervous system. Brain Res 37:21-55.

Desimone R, Gross CG (1979) Visual areas in the temporal cortex of the macaque. Brain Res 178:363-380.

Felleman DJ, Van Essen DC (1991) Distributed hierarchical processing in the primate cerebral cortex. Cerebral Cortex 1:1-47.

Gaffan D, Murray EA (1992) Monkeys with rhinal cortex lesions succeed in object discrimination learning despite 24-hour intervals and fail at matching to sample despite double sample presentation. Behav Neurosci 106:30-38.

Gattas R, Sousa APB, Covey E (1985) Cortical visual areas of the macaque: possible substrates for pattern recognition mechanisms. In: Pattern recognition mechanisms (Chagas C, Gattas R, Gross C, eds), pp 1-20. Pontificae Academiae Scientiarum Scipta Varia No. 54.

Insausti R, Amaral DG, Cowan WM (1987) The entorhinal cortex of the monkey. II. Cortical afferents. J Comp Neurol 264:356-395.

Jones EG (1967) Pattern of cortical and thalamic connexions of the somatic sensory cortex. Nature 216:704-705.

Jones EG, Powell TPS (1970) An anatomical study of converging sensory pathways within the cerebral cortex of the monkey. Brain 93: $793-820$

Kuypers HGJM, Szwarcbart MK, Mishkin M, Rosvold HE (1965) Occipitotemporal corticocortical connections in the rhesus monkey. Exp Neurol 11:245-262.

Leonard BW, Amaral DG, Zola-Morgan S, Squire LR (1992) Selective entorhinal cortical lesions impair learning and memory in the monkey. Soc Neurosci Abstr 18:168.

Martin-Elkins CL, Horel JA (1992) Cortical afferents to behaviorally defined regions of the inferior temporal and parahippocampal gyri as demonstrated by WGA-HRP. J Comp Neurol 321:177-192.

McNaughton BL (1980) Evidence for two physiologically distinct perforant pathways to the fascia dentata. Brain Res 199:1-19.

Meunier M, Murray EA, Bachevalier J, Mishkin M (1990) Effects of perirhinal cortical lesions on visual recognition memory in thesus monkeys. Soc Neurosci Abstr 16:616.

Miller EK, Li L, Desimone R (1991). A neural mechanism for working and recognition memory in inferior temporal cortex. Science 254 : $1377-1379$.

Miller EK, Li L, Desimone R (1993) Activity of neurons in anterior inferior temporal cortex during a short-term memory task. $J$ Neurosci 13:1460-1478.

Milner B (1972) Disorders of learning and memory after temporal lobe lesions in man. Clin Neurosurg 19:421-446.

Milner B, Corkin S, Teuber HL (1968) Further analysis of the hippocampal amnesic syndrome: 14 year follow-up study of H.M. Neuropsychologia 6:215-234.

Mishkin M (1978) Memory in monkeys severely impaired by combined but not separate removal of the amygdala and hippocampus. Nature 273:297-298.

Miyashita X, Chang HS (1988) Neuronal correlate of pictorial shortterm memory in the primate temporal cortex. Nature 331:68-70.

Morris RGM, Garrud P, Rawlins JNP, O'Keefe J (1982) Place navigation impaired in rats with hippocampal lesions. Nature 297:681683.

Murray FA, Mishkin M (1984) Severe tactual as well as visual memory deficits follow combined removal of the amygdala and hippocampus in monkeys. J Neurosci 4:2565-2580.

Murray EA, Mishkin M (1986) Visual recognition in monkeys follow- 
ing rhinal cortical ablations combined with either amygdalectomy or hippocampectomy. J Neurosci 6:1991-2003.

Otto T, Eichenbaum H (1992) Complementary roles of the orbital prefrontal cortex and the perirhinal-entorhinal cortices in an orderguided delayed-nonmatching-to-sample task. Behav Neurosci 106: 763-776.

Riches IP, Wilson FA, Brown MW (1991) The effects of visual stimulation and memory on neurons of the hippocampal formation and the neighboring parahippocampal gyrus and inferior temporal cortex of the primate. J Neurosci 11:1763-1779.

Rockland KS, Pandya DN (1979) Laminar origins and terminations of cortical connections of the occipital lobe in the rhesus monkey. Brain Res 179:3-20.

Room P, Groenewegen HJ (1986) Connections of the parahippocampal cortex. I. Cortical afferents. J Comp Neurol 251:415-450.

Rudy JW, Sutherland RW (1989) The hippocampal formation is necessary for rats to learn and remember configural discriminations. Behav Brain Res 34:97-109.

Saunders RC, Rosene DL (1988) A comparison of the efferents of the amygdala and the hippocampal formation in the rhesus monkey. $I$. Convergence in the entorhinal, prorhinal, and perirhinal cortices. J Comp Neurol 271:153-184.

Scoville WB, Milner B (1957) Loss of recent memory after bilateral hippocampal lesions. J Neurol Neurosurg Psychiatry 20:11-21.

Seltzer B, Pandya DN (1976) Some cortical projections to the parahippocampal area in the rhesus monkey. Exp Neurol 50:146-160.

Squire LR, Haist F, Shimamura AP (1989) The neurology of memory: quantitative assessment of retrograde amnesia in two groups of amnesic patients. J Neurosci 9:828-839.

Steward O (1976) Topographic organization of the projections from the entorhinal areas to the hippocampal formation of the rat. J Comp Neurol 167:285-314.

Suzuki WA, Amaral DG (1990) The organization of cortical inputs to the perirhinal (areas 35 and 36) and parahippocampal (areas TF and TH) cortices in the monkey. Soc Neurosci Abstr 16:53.

Suzuki WA, Zola-Morgan S, Squire LR, Amaral DG (1993) Lesions of the perirhinal and parahippocampal cortices in the monkey produce long-lasting memory impairment in the visual and tactual modalities. J Neurosci 13:2430-2451.

Szabo J, Cowan WM (1984) A stereotaxic atlas of the brain of the cynomolgus monkey (Macaca fascicularis). J Comp Neurol 222:265300.

Tigges J, Spatz WB, Tigges M (1973) Reciprocal point-to-point connections between parastriate and striate cortex in the squirrel monkey (Saimiri). J Comp Neurol 148:481-490.

Tranel D, Brady DR, Van Hoesen GW, Damasio AR (1988) Parahippocampal projections to posterior auditory association cortex (area TPT) in old-world monkeys. Exp Brain Res 70:406-416.
Van Essen DC, Maunsell JHR (1980) Two-dimensional maps of the cerebral cortex. J Comp Neurol 191:225-281.

Van Hoesen GW (1982) The parahippocampal gyrus. New observations regarding its cortical connections in the monkey. Trends Neurosci 5:345-350.

Van Hoesen GW, Pandya DN (1975a) Some connections of the entorhinal (area 28) and perirhinal (area 35) cortices of the rhesus monkey. I. Temporal lobe afferents. Brain Res 95:1-24.

Van Hoesen GW, Pandya DN (1975b) Some connections of the entorhinal (area 28) and perirhinal (area 35) cortices of the rhesus monkey. III. Efferent connections. Brain Res 95:39-59.

Van Hoesen GW, Pandya DN, Butters N (1975) Some connections of the entorhinal (area 28) and perirhinal (area 35) cortices of the rhesus monkey. II. Frontal lobe afferents. Brain Res 95:25-38.

Victor M, Agamanolis J (1990) Amnesia due to lesions confined to the hippocampus: a clinical-pathological study. J Cognit Neurosci 2:246-257.

von Bonin G, Bailey P (1947) The neocortex of Macaca mulatta. Urbana, IL: University of Illinois.

Webster MJ, Ungerleider LG, Bachevalier J (1991) Connections of inferior temporal areas TE and TEO with medial temporal-lobe structures in infant and adult monkeys. J Neurosci 11:1095-1116.

Witter MP, Amaral DG (1991) Entorhinal cortex of the monkey: V. Projections to the dentate gyrus, hippocampus, and subicular complex. J Comp Neurol 307:437-459.

Witter MP, Groenewegen HJ (1984) Laminar origin and septotemporal distribution of entorhinal and perirhinal projections to the hippocampus in the cat. J Comp Neurol 224:371-385.

Witter MP, Van Hoesen GW, Amaral DG (1989) Topographical organization of the entorhinal projection to the dentate gyrus of the monkey. J Neurosci 9:216-228.

Zola-Morgan S, Squire LR (1986) Memory impairment in monkeys following lesions of the hippocampus. Behav Neurosci 100:165-170.

Zola-Morgan S, Squire LR (1990) The primate hippocampal formation: evidence for a time-limited role in memory storage. Science 250: 288-290.

Zola-Morgan S, Squire LR, Amaral DG (1986) Human amnesia and the medial temporal region: enduring memory impairment following a bilateral lesion limited to field CAl of the hippocampus. J Neurosci 6:2950-2967.

Zola-Morgan S, Squire LR, Amaral DG (1989a) Lesions of the hippocampal formation but not lesions of the fornix or the mammillary nuclei produce long-lasting memory impairment in monkeys. J Neurosci 9:897-912.

7ola-Morgan S, Squire LR, Amaral DG, Suzuki WA (1989b) Lesions of perirhinal and parahippocampal cortex that spare the amygdala and hippocampal formation produce severe memory impairment. J Neurosci 9:4355-4370 